ARTICLE

https://doi.org/10.1038/s41467-019-12861-8

\title{
E2F1 acetylation directs p300/CBP-mediated histone acetylation at DNA double-strand breaks to facilitate repair
}

Swarnalatha Manickavinayaham (1) 1, Renier Vélez-Cruz ${ }^{1,3}$, Anup K. Biswas ${ }^{1,4}$, Ella Bedford1, Brianna J. Klein², Tatiana G. Kutateladze (D) ${ }^{2}$, Bin Liu', Mark T. Bedford (10 ${ }^{1} \&$ David G. Johnson (1) ${ }^{1 \star}$

E2F1 and retinoblastoma (RB) tumor-suppressor protein not only regulate the periodic expression of genes important for cell proliferation, but also localize to DNA double-strand breaks (DSBs) to promote repair. E2F1 is acetylated in response to DNA damage but the role this plays in DNA repair is unknown. Here we demonstrate that E2F1 acetylation creates a binding motif for the bromodomains of the p300/KAT3B and CBP/KAT3A acetyltransferases and that this interaction is required for the recruitment of p300 and CBP to DSBs and the induction of histone acetylation at sites of damage. A knock-in mutation that blocks E2F1 acetylation abolishes the recruitment of p300 and CBP to DSBs and also the accumulation of other chromatin modifying activities and repair factors, including Tip60, BRG1 and NBS1, and renders mice hypersensitive to ionizing radiation (IR). These findings reveal an important role for E2F1 acetylation in orchestrating the remodeling of chromatin structure at DSBs to facilitate repair.

\footnotetext{
${ }^{1}$ Department of Epigenetics and Molecular Carcinogenesis, The University of Texas MD Anderson Cancer Center, Science Park, Smithville, TX 78957, USA. ${ }^{2}$ Department of Pharmacology, University of Colorado School of Medicine, Aurora, CO 80045, USA. ${ }^{3}$ Present address: Department of Biochemistry, Midwestern University, Chicago College of Osteopathic Medicine, Downers Grove, IL 60515, USA. ${ }^{4}$ Present address: Herbert Irving Comprehensive Cancer Center, Columbia University, New York, NY 10032, USA. *email: djohnson@mdanderson.org
} 
T he E2F1 transcription factor regulates the expression of genes involved in cell proliferation, apoptosis, and other cellular processes ${ }^{1,2}$. An important regulator of E2F1 is the retinoblastoma $(\mathrm{RB})$ tumor-suppressor protein, which can bind and convert E2F1 from an activator to a repressor of transcription. E2F1 activity is deregulated in most cancers by disruptions in the RB pathway but the role of E2F1 in cancer is complex as it can either promote or inhibit tumor development depending on the context ${ }^{2}$. In addition to regulating transcription, a number of studies have revealed transcription-independent functions for $\mathrm{E} 2 \mathrm{~F} 1$ and $\mathrm{RB}$ in regulating DNA repair ${ }^{3-7}$. E2F1 localizes to sites of DNA damage dependent on its phosphorylation at serine 31 (serine 29 in mice) by the ATM or ATR kinases ${ }^{8,9}$. The topoisomerase II binding protein (TopBP1), which has multiple functions during the DNA damage response, specifically binds this phosphorylated form of E2F1 resulting in recruitment of E2F1 to sites of DNA damage ${ }^{6,8}$. RB associates with E2F1 at DNA double-strand breaks (DSBs) and helps to stabilize the interaction between phosphorylated $\mathrm{E} 2 \mathrm{~F} 1$ and $\mathrm{TopBP} 1^{7}$. Thus there is a mutual dependence between E2F1 and RB for their recruitment to DSBs. Consistent with this finding, the absence of RB causes the same defects in DNA end-resection and homologous recombination (HR) as is observed in the absence of $\mathrm{E}_{2} \mathrm{~F}^{7}$. A knock-in mutation that prevents E2F1 phosphorylation (S29A) and recruitment of E2F1 and $\mathrm{RB}$ to sites of DNA breaks also impairs DNA repair and renders mice hypersensitive to ionizing radiation (IR) ${ }^{7}$.

In addition to phosphorylation, E2F1 is also acetylated in response to DNA damage on three lysine residues (K117, K120, and K125 in human E2F1) near the DNA-binding domain. Different reports have implicated PCAF/KAT2B ${ }^{10,11}$, CBP/KAT3A and $\mathrm{p} 300 / \mathrm{KAT}_{3} \mathrm{~B}^{12,13}$ and Tip60/KAT5 ${ }^{14}$ in mediating E2F1 acetylation. Multiple deacetylases, including histone deacetylase 1 $(\mathrm{HDAC} 1)^{13}$ and SirT $11^{15}$, can also regulate E2F1 acetylation levels. E2F1 acetylation can occur in the absence of E2F1 phosphorylation and independently promotes E2F1 stabilization in response to DNA damage and may also target E2F1 to specific pro-apoptotic genes, such as $p 73^{16}$. Notably, how acetylation regulates the DNA repair function of E2F1 has not been addressed.

Bromodomains specifically recognize and bind acetylated lysine motifs and are found in a number of proteins associated with chromatin and involved in transcriptional regulation and/or DNA repair ${ }^{17}$. Small molecule inhibitors of bromodomains, such as those targeting the BET family of proteins, are emerging as promising drugs for cancers and other diseases ${ }^{17,18}$. It is therefore important to identify the acetylated protein targets that are read and regulated by bromodomain-containing proteins.

Here we demonstrate that the bromodomains of the related p300 and CBP acetyltransferases specifically bind to the acetylated motif of E2F1. This interaction with acetylated E2F1 is critical for p300/CBP recruitment to DSBs and induction of histone acetylation at the sites of damage. Mutating the three sites of E2F1 acetylation also impairs the recruitment of other chromatin-modifying enzymes and repair factors to DSBs, leads to defective DNA repair, and renders mice hypersensitive to IR. These findings define a mechanism by which p300 and CBP are recruited to DSBs dependent on E2F1 and RB and reveal crosstalk between E2F1 acetylation and histone acetylation as an important component of the DNA damage response.

\section{Results}

The bromodomains of p300 and CBP bind to acetylated E2F1. In response to DNA damage, human E2F1 is acetylated on lysine residues K117, K120, and $\mathrm{K} 125^{10,12,14,16}$. To identify proteins that interact with this acetylated form of E2F1, we screened a protein domain microarray with peptides that were either unacetylated or acetylated on lysine residues corresponding to K117, K120, and K125 of human E2F1 (Fig. 1a and Supplementary Fig. 1a). The bromodomain of p300 was identified as binding to the E2F1 peptide when acetylated but not unacetylated (Fig. 1a). Peptide pull-down assays using glutathione S-transferase (GST) fusion proteins confirmed a specific interaction between the acetylated E2F1 peptide and the bromodomain of p300 (Fig. 1b) as well as the bromodomain of the closely related acetyltransferase, CBP (Fig. 1c). The binding was further corroborated by ${ }^{1} \mathrm{H},{ }^{15} \mathrm{~N}$ heteronuclear single quantum coherence (HSQC) nuclear magnetic resonance (NMR) titration experiments (Fig. 1d). The E2F1ac peptide induced chemical shift perturbations in either the isolated bromodomain of p300 or the p300 construct containing bromodomain, the RING finger, and PHD finger (BRP), whereas unacetylated E2F1 peptide failed to do so (Supplementary Fig. 1b). Further analysis using mono-acetylated and di-acetylated E2F1 peptides indicates that acetylation at K125 is sufficient for binding the bromodomains of p300 and CBP (Fig. 1b, c and Supplementary Fig. 1c). However, GST-CBP, but not GST-p300, was also efficiently pulled down using a K117 mono-acetylated peptide (Fig. 1b, c).

E2F1 is recruited to DSB sites via a phospho-specific interaction with the BRCT domain-containing protein TopBP $1^{8}$. We previously used a recombinant GST-TopBP1 fusion protein to specifically pull-down the phosphorylated form of $\mathrm{E} 2 \mathrm{~F} 1$ and its associated proteins ${ }^{7}$. In addition to pulling down E2F1 and RB, the GST-TopBP1 fusion construct also pulled down endogenous p300 and CBP from extracts made from wild-type mouse embryonic fibroblasts (MEFs) treated with IR but not from untreated MEFs (Fig. 2a). A homozygous E2f1 S29A knock-in mutation that prevents E2F1 phosphorylation and its interaction with TopBP1 also prevents association of $\mathrm{p} 300$ and CBP with the GST-TopBP1 fusion construct (Fig. 2a). GST-TopBP1 also pulled down endogenous $\mathrm{p} 300$ and $\mathrm{CBP}$, along with $\mathrm{E} 2 \mathrm{~F} 1$ and $\mathrm{RB}$, from extracts made from human U2OS cells treated with IR but not from untreated cell extract (Fig. 2b). As we previously observed ${ }^{7}$, knocking down RB reduced the interaction between E2F1 and TopBP1 and also prevented the IR-inducible association of p300 and CBP with GST-TopBP1. This suggests that RB helps to stabilize the interaction between $\mathrm{p} 300 / \mathrm{CBP}$ and the phosphorylated form of E2F1 that is recognized by TopBP1.

E2F1 recruits p300 and CBP to DNA DSBs. Previous studies demonstrated that $\mathrm{p} 300$ and $\mathrm{CBP}$ are recruited to DNA breaks and participate in local histone acetylation and remodeling of chromatin structure to facilitate repair ${ }^{19-21}$. However, the mechanism by which p300 and CBP are recruited to DSBs is not fully understood. We previously used an inducible I-PpoI endonuclease system ${ }^{22}$ combined with chromatin immunoprecipitation (ChIP) to demonstrate that $\mathrm{E} 2 \mathrm{~F} 1$ and $\mathrm{RB}$ are enriched at DNA sequences flanking DSBs dependent on E2F1 phosphorylation by $\mathrm{ATM}^{7}$. Using this assay, we confirmed that $\mathrm{E} 2 \mathrm{~F} 1$ and $\mathrm{RB}$ are recruited to an I-PpoI-induced DSB in mouse chromosome 5 (mChrom5) in primary wild-type MEFs but not in E2f1 ${ }^{\text {S29A/S29A }}$ MEFs (Fig. 3a). In contrast, $\gamma \mathrm{H} 2 \mathrm{AX}$ is enriched at the induced DSB in both wild-type and E2f1 ${ }^{S 29 A / S 29 A}$ MEFs. Consistent with our finding that p300 and CBP associate with E2F1 in response to DNA damage, p300 and CBP were also recruited to the induced DSB in wild-type MEFs but not in MEFs harboring the E2f1 S29A mutation (Fig. 3a). Moreover, H3K18ac and $\mathrm{H} 3 \mathrm{~K} 56 \mathrm{ac}$, two histone acetylation marks generated by $\mathrm{p} 300 /$ $\mathrm{CBP}^{23-27}$, were enriched at the DSB in wild-type but not in S29A knock-in MEFs. This defect in p300 and CBP recruitment in 


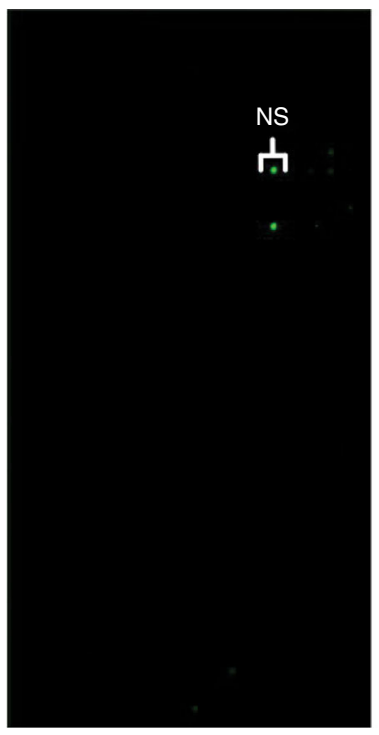

E2F1 UnAc

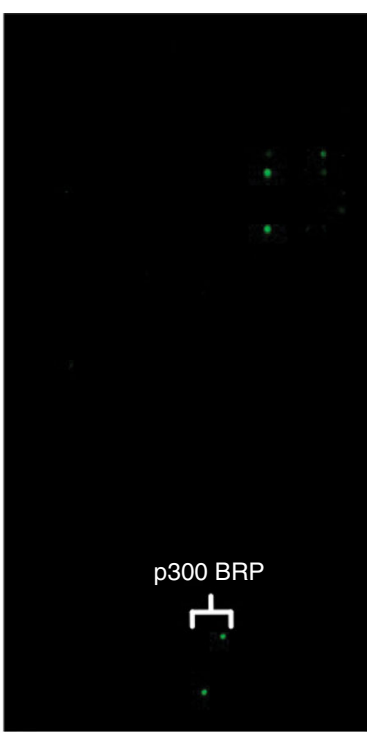

E2F1 3KAc b

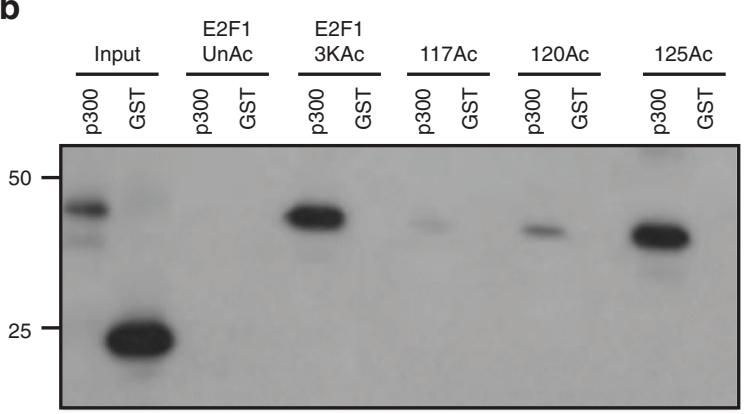

C
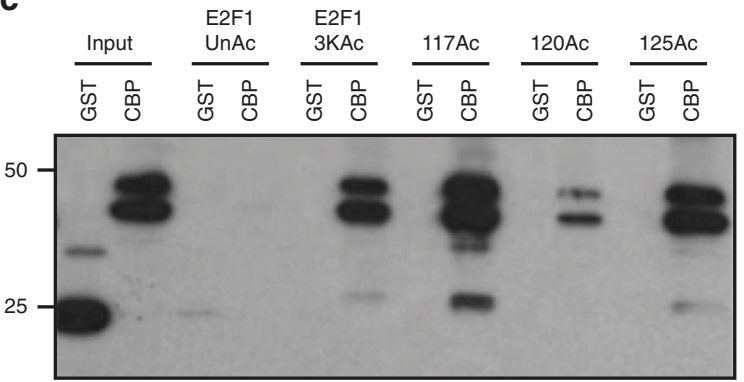

d

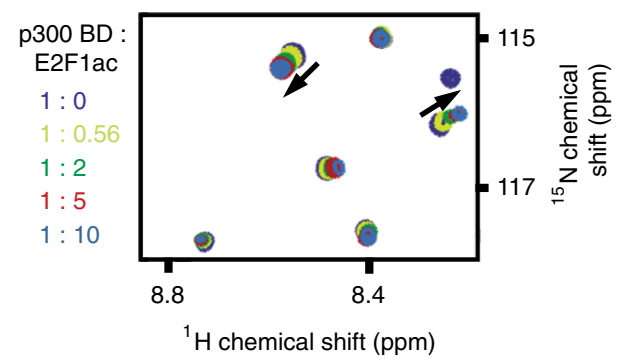

E2f1 ${ }^{S 29 A / S 29 A}$ MEFs is not due to differences in E2F1, RB, p300, or CBP protein levels (Supplementary Fig. 2a). No enrichment of $\mathrm{E} 2 \mathrm{~F} 1, \mathrm{RB}, \mathrm{p} 300, \mathrm{CBP}$, or $\mathrm{H} 3$ acetylation marks was observed at the Gapdh locus, which lacks an I-PpoI cut site (Supplementary Fig. 2b).

The I-PpoI cut site in mChrom5 analyzed above is located in an euchromatic region, 5 ' to RIKEN CDNA 4930519G04 gene. We
Fig. 1 E2F1 acetylation creates a binding motif for the bromodomains of p300 and CBP. a A tri-acetylated E2F1 peptide (3KAc) but not unacetylated peptide (UnAc) binds to recombinant p300 containing the bromo, ring finger, and PHD domains (BRP) in a protein domain microarray. NS indicates nonspecific interaction. b, c E2F1 peptides unacetylated, triacetylated, or mono-acetylated at $\mathrm{K} 117, \mathrm{~K} 120$, or $\mathrm{K} 125$ were incubated with purified GST-fusion proteins containing the bromodomain domain of p300 (b) or CBP (c). Following pull-down, western blot for GST was performed to examine binding between $\mathrm{p} 300$ or $\mathrm{CBP}$ and the E2F1 peptides. $\mathbf{d} \mathrm{A}^{1} \mathrm{H},{ }^{15} \mathrm{~N}$ heteronuclear single quantum coherence NMR titration experiment was performed using the p300 bromodomain and an acetylated E2F1 peptide. Source data of $\mathbf{b}$ and $\mathbf{c}$ are provided as Supplementary Data 5

a
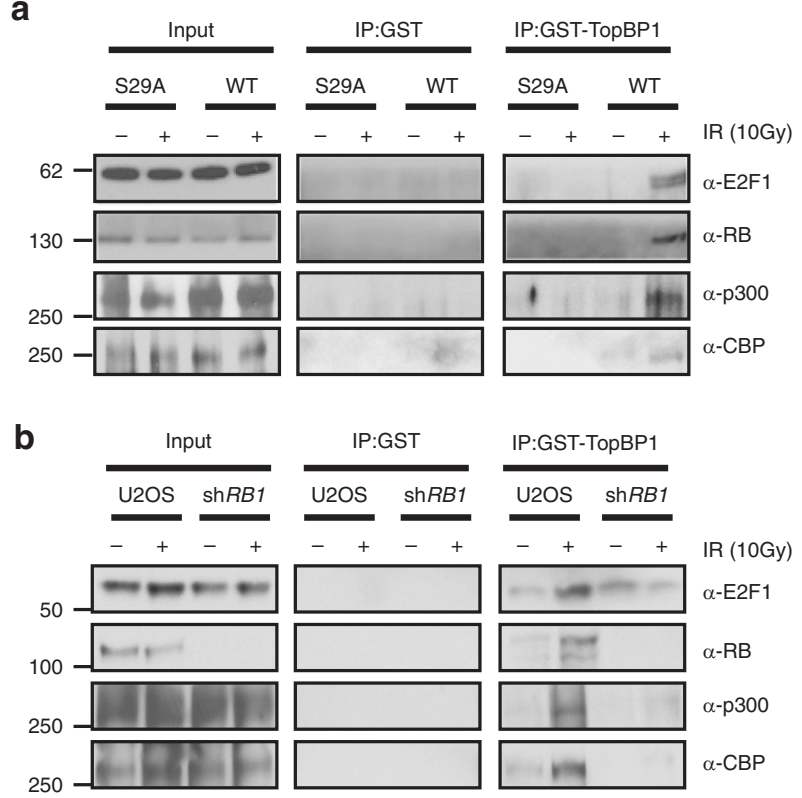

Fig. 2 Phosphorylated E2F1 interacts with $\mathrm{p} 300$ and $\mathrm{CBP}$ in response to DNA damage. a GST-TopBP1 (BRCT1-6) or GST control protein were incubated with whole-cell extract from wild-type (WT) or E2f1529A/S29A (S29A) MEFs that were either untreated ( - ) or treated (+) with IR (10 Gy) and associated proteins were identified $2 \mathrm{~h}$ post-IR by western blot analysis. b A similar GST-TopBP1 pull-down assay was performed using extracts from parental U2OS cells or cells knocked down for RB, either untreated $(-)$ or treated $(+)$ with IR (10 Gy). Source data of $\mathbf{a}$ and $\mathbf{b}$ are provided as Supplementary Data 5

previously demonstrated that $\mathrm{E} 2 \mathrm{~F} 1$ and $\mathrm{RB}$ are also recruited to an I-PpoI-induced DNA break in a heterochromatic region of mouse chromosome $10(\mathrm{mChrom} 10)^{7}$. Both $\mathrm{p} 300$ and CBP were also enriched at this chromosome 10 cut site following I-PpoI induction in wild-type MEFs, as were several $\mathrm{H} 3$ acetylation marks mediated by these enzymes (Supplementary Fig. 2c). However, p300 and CBP recruitment and induction of H3 acetylation at this DSB were abolished in E2f1 ${ }^{S 29 A / S 29 A}$ knock-in MEFs even though $\gamma \mathrm{H} 2 \mathrm{AX}$ was enriched as in wild-type MEFs. Using a similar I-PpoI assay system in human U2OS cells, we find that $\mathrm{E} 2 \mathrm{~F} 1, \mathrm{RB}, \mathrm{p} 300$, and CBP are also recruited to a DSB induced within the DAB1 gene on chromosome $1^{28}$ and this was associated with an induction of H3K56 acetylation (Fig. 3b). Depletion of E2F1 or RB in U2OS cells did not affect p300 or CBP protein levels (Supplementary Fig. 3a) or phospho-ATM enrichment at the DNA break site, but it did prevent enrichment of p300, CBP, and H3K56ac (Fig. 3b). Similar results were observed at the $r D N A$ locus in U2OS cells but not at the control GAPDH locus (Supplementary Fig. 3b, c). Taken together, these findings 
a
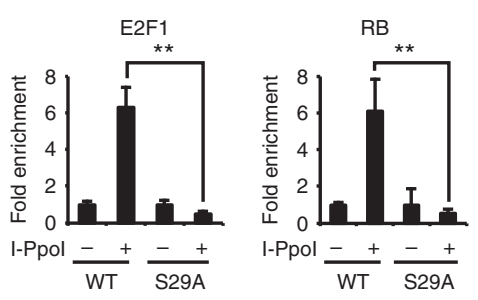

mChrom5 locus
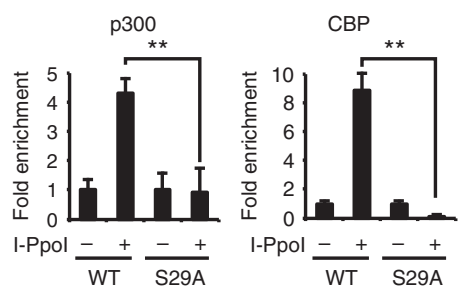
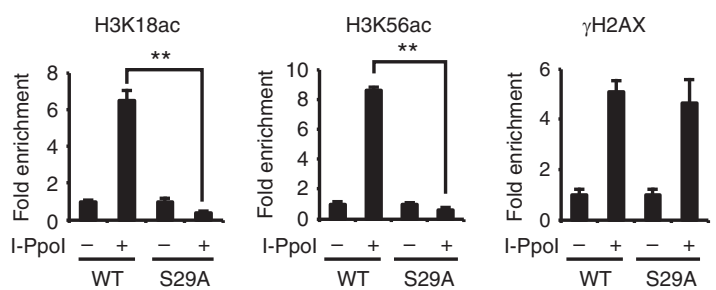

b
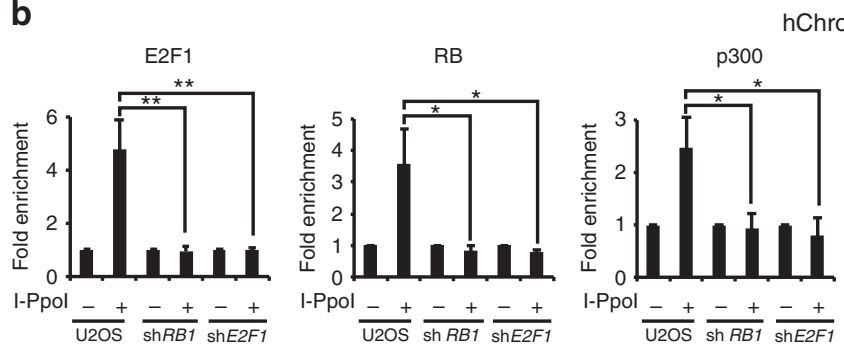

hChrom1 locus
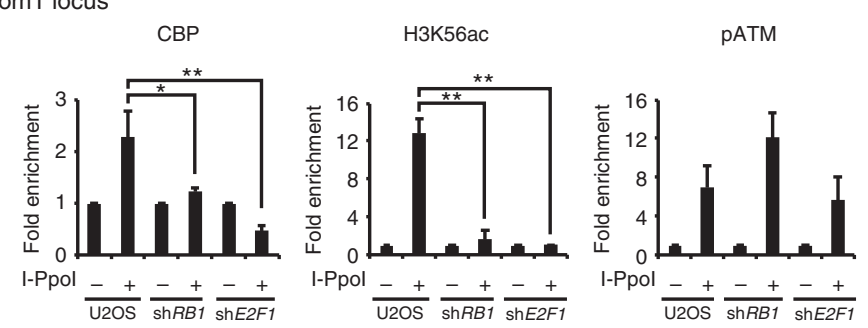

C

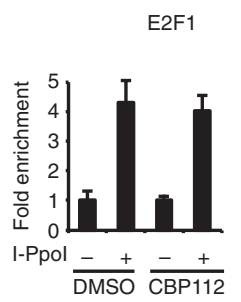

$\mathrm{RB}$

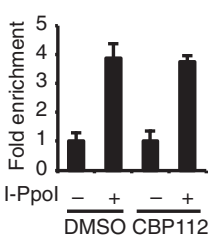

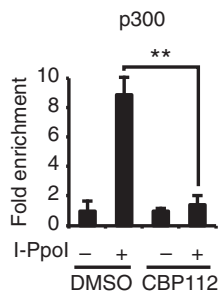

mChrom10 locus
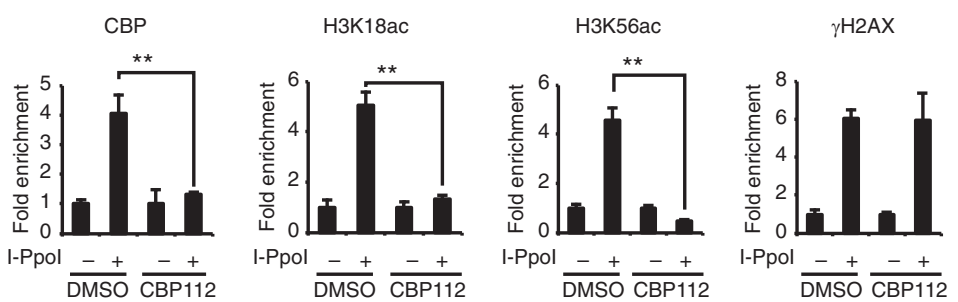

Fig. 3 Recruitment of p300 and CBP to DSBs is dependent on E2F1 and RB. a Primary wild-type (WT) or E2f1S29A/S29A (S29A) MEFs were uninfected ( - ) or infected (+) with a retrovirus expressing HA-ER*-I-Ppol and induced with $2 \mu \mathrm{M}$ 4-hydroxy tamoxifen (4-OHT) for 12 h. ChIP was performed for E2F1, RB, p300, CBP, H3K18ac, H3K56ac, and $\gamma \mathrm{H} 2 \mathrm{AX}$ as indicated. Occupancy was determined by qPCR for a region on mouse chromosome 5 (mChrom5), 166 bp $3^{\prime}$ to an I-Ppol cut site. Fold enrichment represents the ratio between percentage of input of infected cells and uninfected cells. $\mathbf{b} \mathrm{A}$ similar I-Ppol ChIP assay was performed and analyzed as above using parental U2OS cells and U2OS cells expressing shRNA to RB1 and E2F1. Occupancy of E2F1, RB, p300, CBP, H3K56ac, and phospho-ATM was determined for a region on human chromosome 1 (hChrom1), 280 bp 5' to I-Ppol cut site. c Primary wild-type MEFs either untreated (DMSO vehicle) or treated with the bromodomain inhibitor CBP112 ( $1 \mu \mathrm{M}$ for $24 \mathrm{~h}$ ) were subjected to the I-Ppol ChIP assay as above and occupancy was determined by qPCR at the mouse chromosome 10 (mChrom10) locus. Graphs represent average \pm SD of three independent experiments $(n=3)$. $P$ values were calculated by unpaired Student's $t$ test. ${ }^{* \star} P \leq 0.01$ is highly significant and ${ }^{\star} P \leq 0.05$ is significant. Raw data of a-c are provided as Source Data File

reveal that p300 and $\mathrm{CBP}$ are recruited to DSBs in different chromatin environments dependent on E2F1 and RB and that this is critical for inducing several $\mathrm{H} 3$ acetylation marks at the sites of damage.

Incubation of wild-type MEFs with CBP112, a small molecule inhibitor specific for the bromodomains of CBP and $\mathrm{p} 300^{29}$, did not affect $\gamma \mathrm{H} 2 \mathrm{AX}$ induction or recruitment of E2F1 and $\mathrm{RB}$ to an I-PpoI-induced DSB but it did inhibit recruitment of p300 and CBP and induction of H3K18ac and H3K56ac at the site of damage (Fig. 3c). Western blot analysis demonstrated that CBP112 treatment had little effect on the total protein levels of p300 and CBP or occupancy of p300 and CBP at the negative control Gapdh locus (Supplementary Fig. 3d, e). This indicates that the bromodomain inhibitor specifically blocks enrichment of p300 and CBP at DSBs and highlights the importance of the bromodomains for their E2F1-dependent recruitment to DNA breaks.

Generation of E2f1 acetylation-deficient mouse model. We generated a targeted mutant mouse line in which the three sites of E2F1 acetylation were mutated from lysine to arginine and named this allele E2f1 ${ }^{3 K R}$ (Supplementary Fig. $4 \mathrm{a}, \mathrm{b}$ ). Similar to
$E 2 f 1^{-/-}$and $E 2 f 1^{S 29 A / S 29 A}$ knock-in mouse models, homozygous $E 2 f 1^{3 K R / 3 K R}$ knock-in mice are viable and display no obvious phenotype under unstressed conditions. RNA-sequencing (RNAseq) analysis was performed on wild-type and $E 2 f 1^{3 K R / 3 K R} \mathrm{MEFs}$, before and after treatment with the radiomimetic drug neocarzinostatin (NCS), to examine the impact of the $3 \mathrm{KR}$ mutation on global gene expression patterns. Expression levels of E2f1 were similar between wild-type and $E 2 f 1^{3 K R / 3 K R} \mathrm{MEFs}$, consistent with similar E2F1 protein levels in wild-type and 3KR knock-in cells before and after DNA damage (Fig. 4a, b). As expected, the $3 \mathrm{KR}$ mutation prevented E2F1 acetylation in response to DNA damage (Fig. 4c).

A previous study suggested that E2F1 acetylation is involved in regulating the $p 73$ gene promoter in response to DNA damage ${ }^{16}$. In primary MEFs, however, expression of p73 was barely detectable with very few sequence tags in each sample, independent of treatment or genotype (Fig. 4a). In sharp contrast, the expression of Apaf1 and Caspase3, two other pro-apoptotic genes regulated by $\mathrm{E} 2 \mathrm{~F}^{30,31}$, are readily detectable in primary MEFs and their expression increases in response to DNA damage (Fig. 4a). The basal expression level of Apaf1, but not of Caspase3, was lower in $E 2 f 1^{3 K R / 3 K R} \mathrm{MEFs}$, but induction in response to DNA damage was similar between genotypes for both genes, and 
a

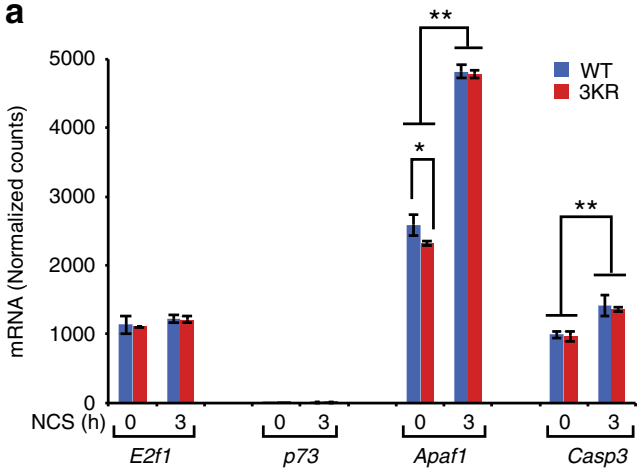

d

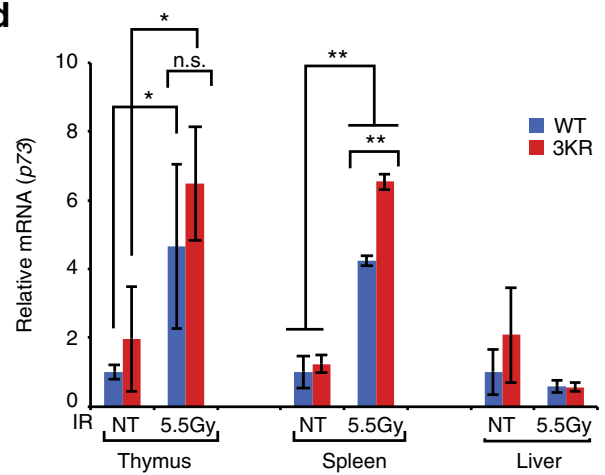

b
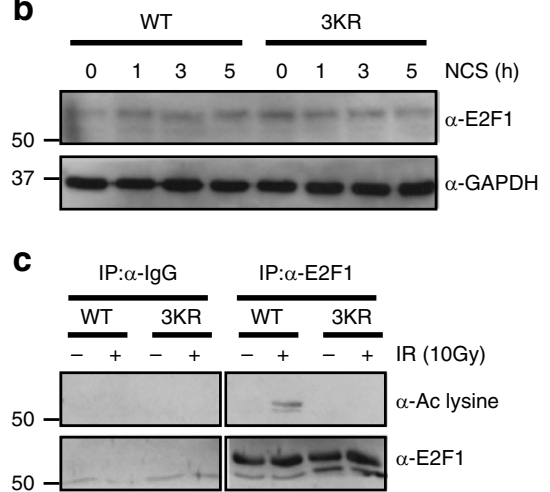

e

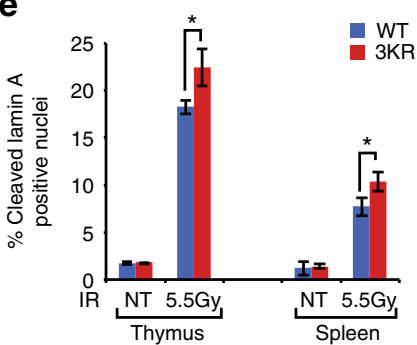

Fig. 4 Characterization of the E2F1 acetylation 3KR knock-in mouse model. a RNA-seq was performed on wild-type (WT) and E2f13KR/3KR (3KR) MEFs untreated or treated with NCS (250 ng/ml for $3 \mathrm{~h}$ ) and the normalized mRNA counts for E2f1, p73, Apaf1, and Caspase3 (Casp3) are presented. b Western blot analysis was performed to examine E2F1 protein levels in primary wild-type (WT) and E2f13KR/3KR (3KR) MEFs before and after DNA damage with NCS $(250 \mathrm{ng} / \mathrm{ml})$ for the indicated times. c Wild-type and E2f13KR/3KR MEFs were untreated or exposed to $10 \mathrm{~Gy} I \mathrm{R}$ and cell extracts made $2 \mathrm{~h}$ later. Coimmunoprecipitation was performed using control IgG or antibody to E2F1 (C-20, rabbit polyclonal) followed by western blot analysis for acetylated lysine (top) or E2F1 (bottom) using a different antibody (KH95, mouse monoclonal). d Wild-type (WT) and E2f13KR/3KR (3KR) mice were exposed to 5.5 Gy of IR and tissues collected $2 \mathrm{~h}$ later. Total RNA was isolated and cDNA was prepared by RT-PCR using random hexamer primer. Expression of $p 73$ in the thymus, spleen, and liver was determined by $9 P C R$. e Wild-type (WT) and E2f13KR/3KR (3KR) mice were exposed to $5.5 \mathrm{~Gy}$ of IR and thymus and spleen tissues were collected $2 \mathrm{~h}$ later. Tissue sections were stained for the cleaved form of Lamin A. The average of percentage of positive nuclei is presented. All graphs represent average $\pm \mathrm{SD}$ of three independent experiments $(n=3)$ of cells or mice. $P$ values were calculated by unpaired Student's $t$ test. ${ }^{\star \star} P \leq 0.01$ is highly significant and ${ }^{\star} P \leq 0.05$ is significant. Source data of $\mathbf{b}$ and $\mathbf{c}$ are provided as Supplementary Data 5. Raw data of $\mathbf{a}, \mathbf{d}$, and $\mathbf{e}$ are in Source Data File

this was confirmed by reverse transcriptase real-time quantitative PCR (RT-qPCR) analysis of independent samples (Supplementary Fig. 4c). To determine whether $p 73$ expression and induction in response to DNA damage is tissue restricted, $E 2 \mathrm{fl}^{3 K R / 3 K R}$ and wild-type control mice were untreated or exposed to $5.5 \mathrm{~Gy}$ of IR and RNA was isolated from various tissues $2 \mathrm{~h}$ post-IR. The expression of $p 73$ was significantly induced in the thymus and spleen, but not in the liver of irradiated mice. However, contrary to expectations, the E2F1 3KR mutation actually enhanced the induction of $p 73$ expression in response to IR, although this was significant only in the spleen (Fig. 4d). Consistent with this finding, the apoptotic response to IR in the thymus and spleen was higher in $E 2 f 1^{3 K R / 3 K R}$ mice compared to wild-type mice (Fig. 4e).

Gene Set Enrichment Analysis (GSEA) was used to analyze the RNA-seq data from primary MEFs to determine which functional pathways might be altered by the E2F1 3KR mutation. Fifty-five out of 2499 gene sets were significantly enriched (upregulated) in wild-type cells compared to $E 2 f 1^{3 K R / 3 K R}$ mutant cells before DNA damage (Supplementary Data 1) and 96 gene sets were enriched in wild-type cells compared to $E 2 f 1^{3 K R / 3 K R}$ cells after DNA damage (Supplementary Data 2). The majority of gene sets whose expression was significantly lower in E2f $13 K R / 3 K R$ mutant cells compared to wild-type cells, either before or after DNA damage, were related to neurodevelopment and/or differentiation. Almost 200 gene sets were found to be significantly enriched in $E 2 f 1^{3 K R /}$
$3 K R$ cells compared to that in wild-type cells in the absence or presence of DNA damage, respectively. Gene sets upregulated in $E 2 f 1^{3 K R / 3 K R}$ cells were primarily involved in innate immune function (Supplementary Data 3 and 4). Importantly, no gene sets related to DNA repair, DNA damage response, cell cycle, or apoptosis were significantly different between wild-type and E2f1 ${ }^{3 K R / 3 K R}$ knock-in MEFs, before or after DNA damage. These findings indicate that, while the E2F1 3KR mutation has a significant impact on global gene expression patterns, even in the absence of DNA damage, genes involved in DNA damage repair and cell cycle checkpoint responses are not significantly affected.

E2F1 3KR mutation impairs histone acetylation at DSBs. To determine how mutating the sites of E2F1 acetylation impacts its recruitment to DSBs, as well as the recruitment of other factors, we performed the I-PpoI ChIP assay in primary MEFs derived from homozygous $E 2 f^{3 K R / 3 K R}$ knock-in and wild-type control mice. The E2F1 3KR mutation did not significantly impact induction of $\gamma \mathrm{H} 2 \mathrm{AX}$ or enrichment of E2F1 and RB at a DSB located on mChrom 10 but recruitment of p300 and CBP and induction of H3K18ac and H3K56ac were abolished (Fig. 5a). This difference in recruitment was not due to differences in p300 and CBP protein levels between genotypes before or after DNA damage induction (Fig. 5b). Similar results were obtained at another I-PpoI cut site on mChrom5 (Supplementary Fig. 5a). No 
mChrom10 locus
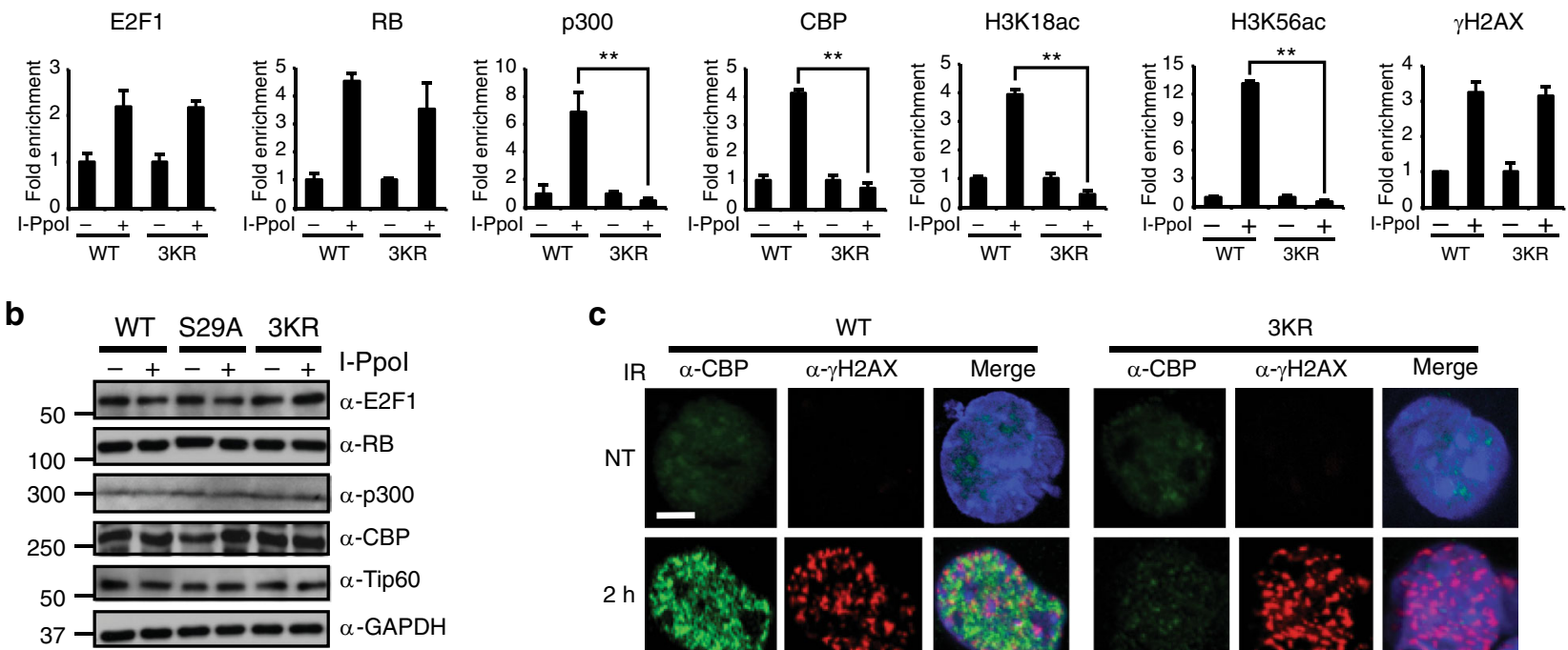

C
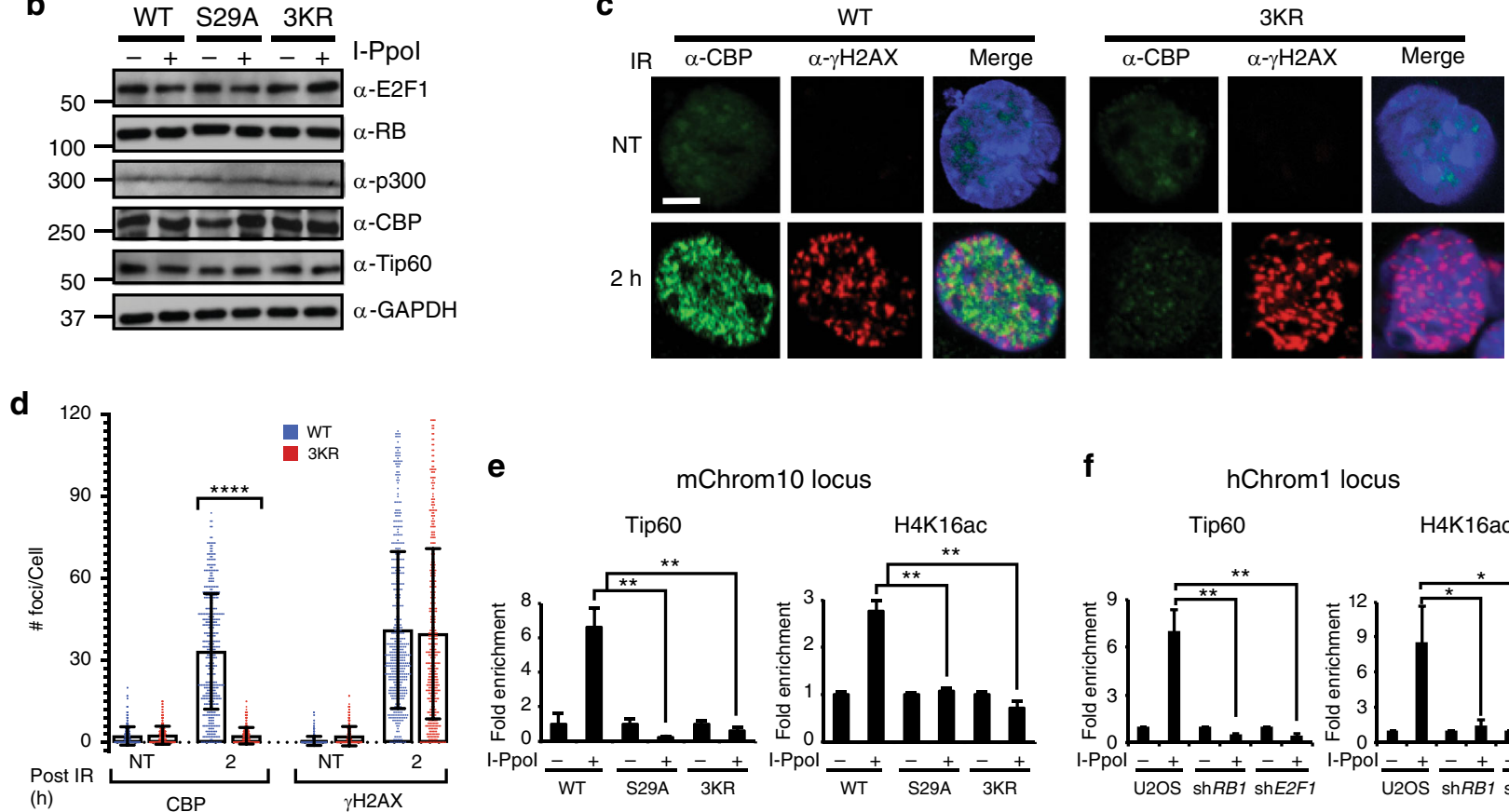

f

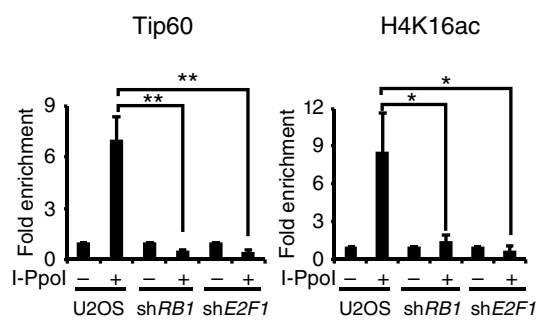

Fig. 5 The E2F1 3KR mutation impairs multiple histone acetylation events at DSBs. a Primary wild-type (WT) and E2f13KR/3KR (3KR) MEFs were subjected to the I-Ppol ChIP assay and qPCR was performed to determine the occupancy of the indicated proteins and $\mathrm{H} 3$ acetylation marks at mChrom10 locus. b Wild-type (WT), E2f1529A/S29A (S29A), and E2f13KR/3KR (3KR) MEFs were uninfected or infected with a retrovirus expressing inducible I-Ppol, treated with 4-OHT, and western blot analysis was performed for E2F1, RB, p300, CBP, Tip60, and GAPDH. c Wild-type and E2f13KR/3KR MEFs were mock treated (NT) or exposed to $5 \mathrm{~Gy}$ of IR $2 \mathrm{~h}$ prior to in situ extraction and fixation. Representative images show formation of IR-induced foci for CBP (green) and $\gamma \mathrm{H} 2 \mathrm{AX}$ (red) and merged with DAPI staining (blue) of nuclei. Bar, $10 \mu \mathrm{m}$. d Quantification of CBP and $\gamma \mathrm{H} 2 \mathrm{AX}$ foci. Each treatment group was in triplicate, and in total at least 450 cells $(n>450)$ were counted per treatment group. Graphs represent average \pm SD of foci per cell. Overlaid scattered dot plot shows the distribution of foci. $P$ values were calculated by unpaired Mann-Whitney $U$ test. ${ }^{\star \star \star \star} P<0.0001$ is highly significant. e Primary wild-type (WT), E2f1S29A/S29A (S29A), and E2f13KR/3KR (3KR) MEFs were subjected to the I-Ppol ChIP assay and occupancy of Tip60 and H4K16ac at mChrom10 locus was determined. $\mathbf{f}$ Parental U2OS cells and U2OS cells expressing shRNA to RB1 and E2F1 were subjected to the I-Ppol ChIP assay and qPCR was performed to determine occupancy of Tip60 and H4K16ac at hChrom1 locus. Except $\mathbf{d}$, other graphs represent average \pm SD of three independent experiments ( $n=3$ ). $P$ values were calculated by unpaired Student's $t$ test. ${ }^{\star \star} P \leq 0.01$ is highly significant and ${ }^{\star} P \leq 0.05$ is significant. Source data of $\mathbf{b}$ is provided as Supplementary Data 5. Raw data of $\mathbf{a}$ and $\mathbf{d}-\mathbf{f}$ are in Source data file

enrichment of $\mathrm{p} 300, \mathrm{CBP}$, or histone $\mathrm{H} 3$ acetylation marks was observed in either MEF culture after I-PpoI induction at the negative control Gapdh locus (Supplementary Fig. 5b). These findings are consistent with the bromodomain inhibitor data and the model in which phosphorylation recruits $\mathrm{E} 2 \mathrm{~F} 1$ and $\mathrm{RB}$ to DNA breaks while E2F1 acetylation is recognized by p300 and $\mathrm{CBP}$ leading to their recruitment and induction of $\mathrm{H} 3$ acteylation at the sites of DNA damage.

To further support this model, immunofluorescence (IF) for CBP and p300 was performed to observe IR-induced foci formation as an indicator of recruitment to DSBs. CBP and p300 both formed foci in response to IR that partially co-localized with $\gamma \mathrm{H} 2 \mathrm{AX}$ in wild-type MEFs (Fig. 5c, d and Supplementary Fig. 5c). However, the E2F1 3KR mutation abolished IR-induced foci formation of p300 and CBP while $\gamma \mathrm{H} 2 \mathrm{AX}$ induction was unaffected. These findings again indicate an important function for E2F1 acetylation in the recruitment of $\mathrm{p} 300 / \mathrm{CBP}$ to damaged DNA.

In addition to $\mathrm{H} 3$ acetylation, histone $\mathrm{H} 4$ acetylation is also known to be induced at DSBs by the Tip60 acetyltransferase and this is important for DNA repair and cell cycle checkpoint signaling ${ }^{20,32-34}$. As expected, ChIP analysis revealed Tip60 and H4K16ac enrichment at an I-PpoI-induced break on mChrom10 in wild-type MEFs (Fig. 5e). However, mutating the sites of E2F1 phosphorylation (S29A) or acetylation (3KR) prevented Tip60 recruitment and $\mathrm{H} 4 \mathrm{~K} 16 \mathrm{ac}$ induction at the DSB site (Fig. 5e). Similar results were observed at another I-PpoI-induced cut site on mChrom5 but not at the negative control Gapdh locus (Supplementary Fig. 6a, b).

Depleting E2F1 or RB in U2OS cells also impaired Tip60 recruitment and induction of $\mathrm{H} 4 \mathrm{~K} 16 \mathrm{ac}$ at I-PpoI-induced DNA breaks on human chromosome 1 and the $r D N A$ locus (Fig. $5 \mathrm{f}$ and 
Supplementary Fig. 6c) but not at the GAPDH locus (Supplementary Fig. 6d). Differences in Tip60 protein levels were not responsible for the defects observed in Tip60 recruitment when E2F1 was mutated or when E2F1 and RB were depleted (Fig. 5b and Supplementary Fig. 3a). Unlike p300 and CBP, no association was observed between Tip60 and phosphorylated E2F1 in the GST-TopBP1 pull-down assay (Supplementary Fig. 6e). This suggests that E2F1 may regulate Tip60 recruitment or retention at DNA breaks and induction of $\mathrm{H} 4$ acetylation through an indirect mechanism.

The 3KR mutation prevents BRG1 and MRN accumulation at DSBs. Our previous studies demonstrated that E2F1 and RB are required for the recruitment of a BRG1-containing nucleosome remodeling complex to DSBs and for decreasing nucleosome density at the sites of damage ${ }^{7}$. To determine whether E2F1 acetylation impacts this process, E2f1 ${ }^{3 K R / 3 K R}$ knock-in MEFs and the I-Ppo-I ChIP assay system were employed. The E2F1 3KR mutation did not affect BRG1 protein levels (Supplementary Fig. 7a), but it did impair BRG1 recruitment to a DNA break (Fig. 6a). As we previously observed ${ }^{7}$, E2F1-dependent recruitment of BRG1 is associated with decreased levels of total $\mathrm{H} 3$ protein at the site of damage, despite the increased levels of $\mathrm{H} 3$ acetylation marks (Fig. 6a).

It has been suggested that BRG1-containing complexes are recruited to DSBs through binding of the BRG1 bromodomain to acetylated histone $\mathrm{H} 3$ motifs $^{19}$. Thus E2F1 acetylation may regulate BRG1 recruitment to DNA breaks by indirectly promoting p300/CBP-mediated H3 acetylation. On the other hand, we previously found that BRG1 physically associates with phosphorylated $\mathrm{E} 2 \mathrm{~F} 1$ and $\mathrm{RB}$ in response to DNA damage, suggesting a direct role for E2F1 in recruiting BRG1 to sites of DNA breaks ${ }^{7}$. To differentiate between these mechanisms, the GST-TopBP1 pull-down assay was performed using $E 2 f 1^{3 K R / 3 K R}$ knock-in and wild-type control MEF extracts. The $3 \mathrm{KR}$ mutation did not prevent GST-TopBP1 from associating with E2F1 and RB in response to DNA damage, indicating that $\mathrm{E} 2 \mathrm{~F} 1$ phosphorylation and its interactions with TopBP1 and RB are unaffected by the 3KR knock-in mutation (Fig. 6b). As expected, the E2F1 3KR mutation prevented association of p300 with the GST-TopBP1E2F1 complex (Fig. 6b). The ability of GST-TopBP1 to pull-down BRG1 was also compromised by the $3 \mathrm{KR}$ mutation, suggesting that E2F1 acetylation stabilizes the interaction between BRG1 and E2F1-RB (Fig. 6b). Indeed, BRG1 is known to physically interact with p300 in the context of transcription of E2F target genes ${ }^{35}$.

The Mre11-RAD50-NBS1 (MRN) complex is a key DNA damage sensor and has roles in DNA end processing and in promoting the recruitment of various DNA repair proteins and chromatin-modifying activities to sites of damage. We previously observed that the absence of E2F1 impaired NBS1 foci formation in response to IR, suggesting that E2F1 is important for the recruitment and/or retention of $\mathrm{MRN}$ at $\mathrm{DSBs}^{4}$. Indeed, NBS1 and Mre11 were both enriched at an I-PpoI-induced DNA break in wild-type MEFs but not in E2f1 ${ }^{S 29 A / S 29 A}$ or E2f1 ${ }^{3 K R / 3 K R}$ MEFs (Fig. 6c). The E2F1 knock-in mutations did not affect the protein levels of Mre11 or NBS1 before or after DNA damage (Supplementary Fig. 7b). These findings confirm a role for E2F1 in the recruitment and/or retention of MRN at DNA breaks and indicates that both E2F1 phosphorylation and acetylation are important for this process.

NBS1 and Tip60 each participate in the activation of ATM at the sites of DNA damage $33,36-38$. Given that E2F1 and its a

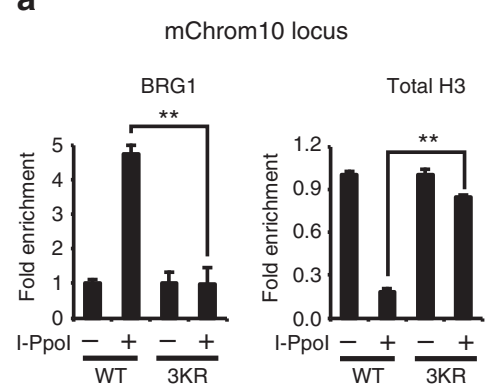

b

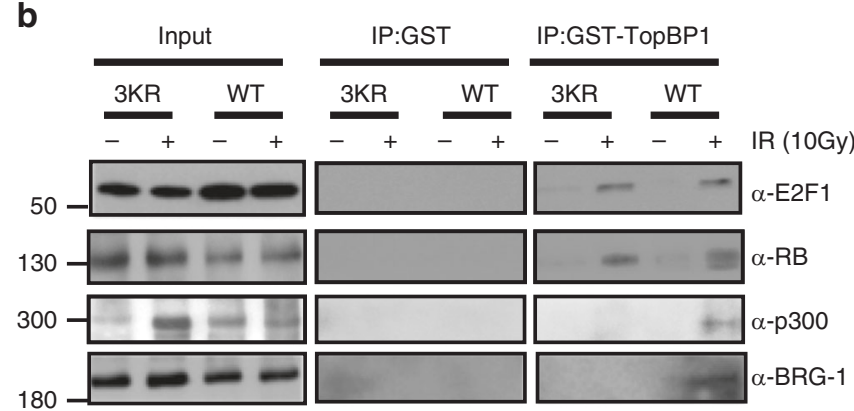

C

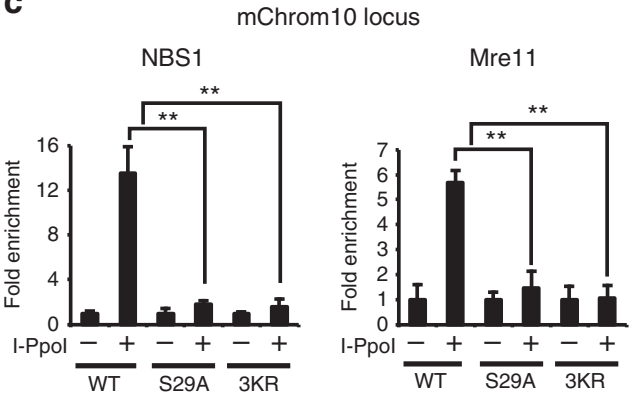

d

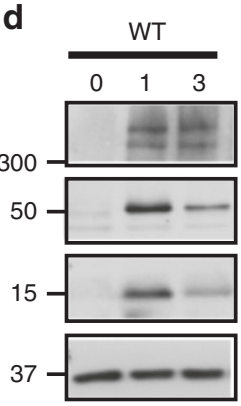

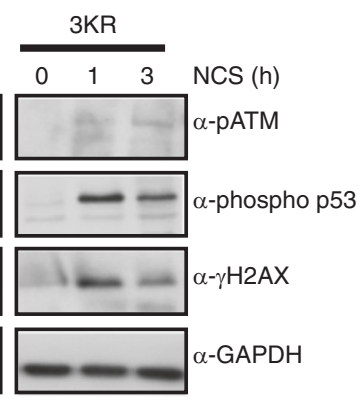

Fig. 6 The E2F1 3KR mutation impairs BRG1 and MRN accumulation at a DSB. a Primary wild-type (WT) and E2f73KR/3KR (3KR) MEFs were subjected to the I-Ppol ChIP assay and occupancy of BRG1 and total histone H3 was determined by qPCR at mChrom10 locus. b Purified GST-TopBP1 (BRCT1-6) or GST control protein was incubated with whole-cell extract from wild-type (WT) or E2f13KR/3KR (3KR) MEFs that were either untreated ( - ) or treated $(+)$ with IR $(10 \mathrm{~Gy})$ and harvested $2 \mathrm{~h}$ post-IR. Associated proteins were pulled down using glutathione beads and western blot was performed with the indicated antibodies. c Primary wild-type (WT), E2f1S29A/S29A (S29A), and E2f13KR/3KR (3KR) MEFs were subjected to the I-Ppol ChIP assay and occupancy of NBS1 and Mre11 was determined by qPCR at mChrom10 locus. d Western blot analysis was performed for phospho-ATM (serine 1981), phospho-p53 (serine 15), $\gamma \mathrm{H} 2 \mathrm{AX}$, and GAPDH using whole-cell extracts from primary wild-type (WT) and E2f13KR/3KR (3KR) MEFs, before treatment or $1 \mathrm{~h}$ and $3 \mathrm{~h}$ post-treatment with $250 \mathrm{ng} / \mathrm{ml}$ of NCS. All graphs represent average \pm SD of three independent experiments $(n=3)$. $P$ values were calculated by unpaired Student's $t$ test. ${ }^{\star \star} P \leq 0.01$ is highly significant. Source data of $\mathbf{b}$ and $\mathbf{d}$ are provided as Supplementary Data 5. Raw data of $\mathbf{a}$ and $\mathbf{c}$ are in Source Data File 
posttranslational modifications are required for the accumulation of both factors at DSBs, it might be expected that ATM activation would be impaired by the E2F1 3KR knock-in mutation. As predicted, autophosphorylation of ATM at serine 1981, a marker of ATM activation ${ }^{39}$, was significantly reduced in response to DNA damage in $E 2 f 1^{3 K R / 3 K R}$ mutant cells compared to wild-type cells although induction of $\mathrm{p} 53$ phosphorylation at serine 15 and $\mathrm{H} 2 \mathrm{AX}$ phosphorylation at serine $139(\gamma \mathrm{H} 2 \mathrm{AX})$ was not compromised by the $3 \mathrm{KR}$ knock-in mutation (Fig. 6d). This suggests that low levels of active ATM, or kinases related to ATM, are sufficient to induce normal levels of p53 and H2AX phosphorylation in E2f1 $1^{3 K R / 3 K R}$ cells. Together, our results indicate that both E2F1 phosphorylation and acetylation are important for the recruitment of nucleosome remodeler BRG1 and MRN complex to DSBs.

The E2F1 3KR knock-in mutation causes defective DSB repair. As a read-out of DSB repair competency, we measured the kinetics of $\gamma \mathrm{H} 2 \mathrm{AX}$ and 53BP1 foci formation and clearance following IR exposure in wild-type and $E 2 f 1^{3 K R / 3 K R}$ MEFs. The $3 K R$ mutation does not significantly affect $\gamma \mathrm{H} 2 \mathrm{AX}$ foci formation at earlier time points post-irradiation, but $\gamma \mathrm{H} 2 \mathrm{AX}$ foci clearance is significantly impaired at later time points in the knock-in compared to wild-type MEFs (Fig. 7a, b). Likewise, IR-induced 53BP1 foci formation in $E 2 f 1^{3 K R / 3 K R}$ MEFs is comparable to wild-type MEFs at early time points but 53BP1 foci clearance at later time points is significantly delayed (Fig. $7 \mathrm{a}, \mathrm{c}$ ). These findings suggest that the initial response to IR-induced DSBs is unaffected by the E2F1 3KR mutation but that later steps in the DSB repair process are impaired.

Cells defective for DSB repair are sensitized to chromosomal aberrations under genotoxic stress, which can lead to genome instability. To examine the impact of the E2F1 3KR mutation on chromosome maintenance, metaphase spreads were prepared from wild-type and $E 2 f 1^{3 K R / 3 K R}$ MEFs, 48 h post-IR. As expected, $E 2 f 1^{3 K R / 3 K R}$ MEFs had a significant increase in the percentage of cells with chromosome fusions, including dicentrics, rings, acentric, and long acrocentric chromosome-type aberrations (Fig. 7 d). In addition, E2f1 ${ }^{3 K R / 3 K R}$ metaphase spreads displayed increased numbers of small fragments and tetra/polyploid cells, which account for the significantly higher aberrant metaphases in the knock-in MEFs compared to wild-type MEFs (Fig. 7d). Of note, similar phenotypes of delayed DNA damage clearance and increased chromosome aberrations in response to genotoxic stress are also observed in E2f1 ${ }^{S 29 A / S 29 A}$ cells and in cells lacking E2F1 or $\mathrm{RB}^{4,7}$.

We previously demonstrated that E2f1 $229 A / S 29 A$ mice are hypersensitive to IR, a hallmark of defective DSB repair ${ }^{7}$. To determine the functional significance of E2F1 acetylation in vivo, $E 2 f 1^{3 K R / 3 K R}$ and wild-type sibling control mice were irradiated with $5.5 \mathrm{~Gy}$ of IR and maintained under sterile conditions. The majority of $E 2 f 1^{3 K R / 3 K R}$ mice did not survive beyond 35 days following IR exposure, while $80 \%$ of wild-type siblings survived with no apparent signs of ill-health (Fig. 7e). Thus, like E2F1 phosphorylation, E2F1 acetylation may also play an important role in maintaining organismal homeostasis and survival in response to IR.

\section{Discussion}

Prior studies have indicated a role for E2F1 acetylation in regulating its transcriptional activity, particularly the induction of $p 73$ gene expression in response to DNA damage ${ }^{11,13,16}$. However, in primary MEF cultures we find that $p 73$ gene expression is barely detectable and unresponsive to DNA damage. On the other hand, $p 73$ expression was induced in response to DNA damage in several mouse tissues, although mutation of the three sites of acetylation in E2F1 either had no significant impact or actually enhanced this induction.

To gain a better understanding of how acetylation may regulate E2F1 transcriptional activity, we performed GSEA on RNA-seq data from primary MEFs derived from $E 2 f 1^{3 K R / 3 K R}$ and wild-type control mice, before and after the induction of DNA damage. Unexpectedly, $>200$ gene sets were significantly different between wild-type and $E 2 f 1^{3 K R / 3 K R}$ cells even in the absence of DNA damage. Gene sets that were enriched in wild-type cells compared to $E 2 f 1^{3 K R / 3 K R}$ cells, and thus likely to be positively regulated by E2F1 acetylation, were primarily related to nervous system development and differentiation (Supplementary Data 1 and 2). Importantly, gene sets related to DNA repair, DNA damage response signaling, cell proliferation, cell cycle checkpoints, or apoptosis were not significantly different between wild-type and $E 2 f 1^{3 K R / 3 K R}$ knock-in primary MEFs. This suggests that impaired DNA repair in $E 2 f 1^{3 K R / 3 K R}$ mutant MEFs, as indicated by delayed $\gamma \mathrm{H} 2 \mathrm{AX}$ and 53BP1 foci clearance and increased chromosomal aberrations following IR exposure, is not due to an indirect effect on transcription but rather on a direct effect of E2F1 at DSB sites. Interestingly, gene sets enriched in $E 2 f 1^{3 K R / 3 K R}$ MEFs were primarily involved in innate immunity and response to pathogens (Supplementary Data 3 and 4). Whether this is due to a direct transcriptional effect or an indirect consequence of genome instability caused by the E2f1 knock-in mutation ${ }^{40}$ is at present unclear.

E2F1 is recruited to DNA damage through a phospho-specific interaction with one of the BRCT domains of TopBP $1^{8}$. We previously demonstrated that the $\mathrm{RB}$ tumor-suppressor protein associates with this phosphorylated form of E2F1 and is required for the stable interaction between E2F1 and TopBP1 ${ }^{7}$. E2F1 and $\mathrm{RB}$ in turn recruit a BRG1-containing SWI/SNF complex to DSBs and this is associated with a decrease in total nucleosome density at the sites of damage ${ }^{7}$. We now show that an interaction between acetylated E2F1 and the bromodomains of p300 and CBP allows E2F1 and RB to directly recruit p300/CBP to sites of DNA breaks. Our data indicate that p300 and CBP then mediate the acetylation of multiple lysine residues on histone $\mathrm{H} 3$, including $\mathrm{H} 3 \mathrm{~K} 18$ and $\mathrm{H} 3 \mathrm{~K} 56$, in nucleosomes flanking DSBs. E2F1 and RB are also required for the recruitment of Tip60 and induction of $\mathrm{H} 4 \mathrm{~K} 16 \mathrm{ac}$ at DNA breaks, although this may involve an indirect mechanism rather than a direct interaction between Tip60 and E2F1 or RB (Fig. 8).

Roles for p300, CBP, and Tip60 in the repair of DSBs are well established ${ }^{19-21,23,27,32,33,41}$. The p300 and CBP proteins were previously shown to localize to DSBs and to be required for H3K18 acetylation and the recruitment of SWI/SNF to sites of damage ${ }^{20,21}$. Knocking down p300 and CBP was also shown to impair $\mathrm{H} 4$ acetylation, the recruitment of DNA repair proteins to sites of damage, and DNA repair efficiency by both HR and nonhomologous end-joining (NHEJ) pathways ${ }^{20,21,25}$. Findings presented here are consistent with those previous studies and now establish the molecular mechanism by which p300 and CBP are recruited to sites of DSBs dependent on RB and E2F1 posttranslational modifications. This role for E2F1 in recruiting histone acetyltransferases to regulate DNA repair is reminiscent of its function in activating transcription, although the mechanisms by which E2F1 localizes to sites of DNA damage and target gene promoters are different ${ }^{42-45}$.

Studies in the 1970s and 1980s demonstrated that histone acetylation and nucleosome remodeling occurs during the process of nucleotide excision repair (NER) ${ }^{46,47}$. Indeed, we previously demonstrated a role for E2F1 in the induction of histone acetylation and chromatin decondensation in response to ultraviolet radiation to promote efficient $\mathrm{NER}^{3,48}$. More recent studies are 
a

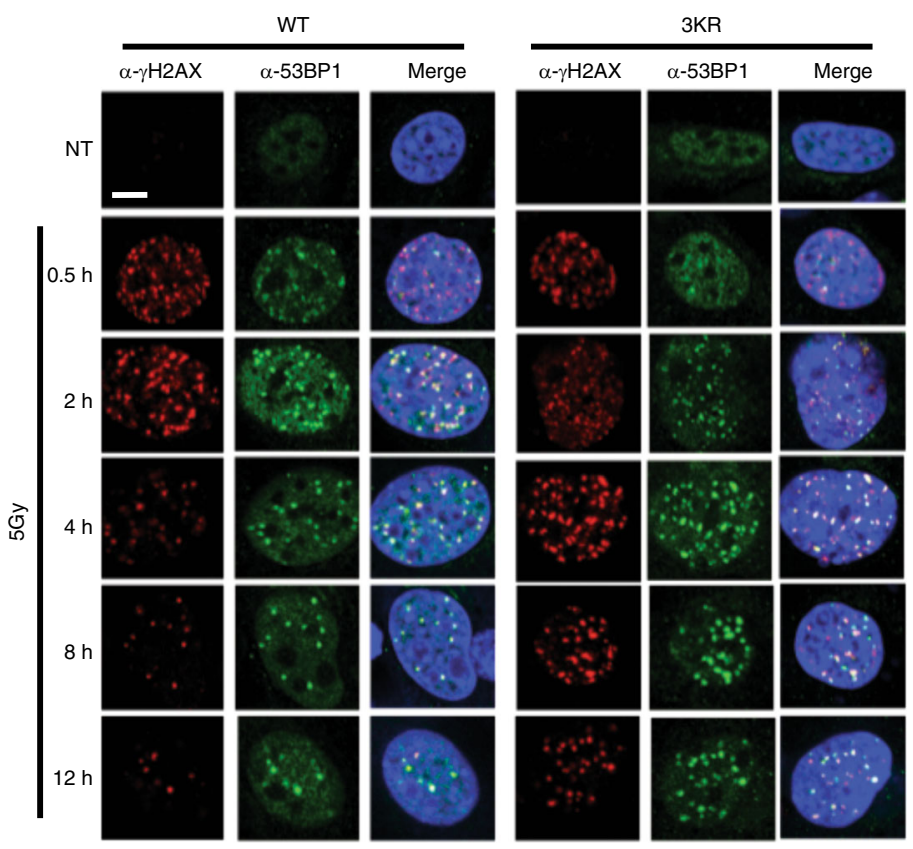

b

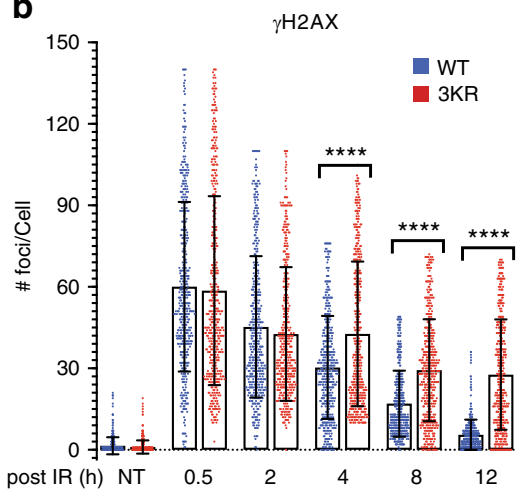

C

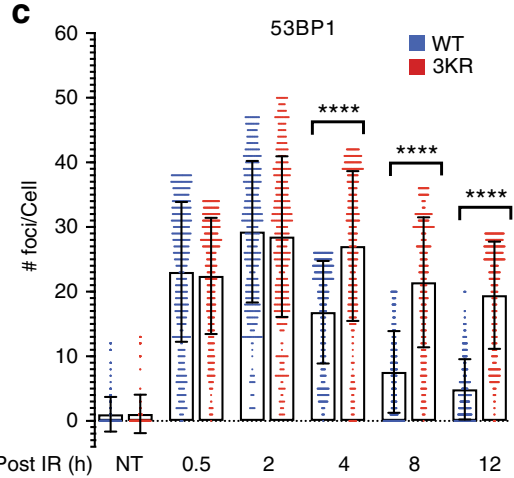

d

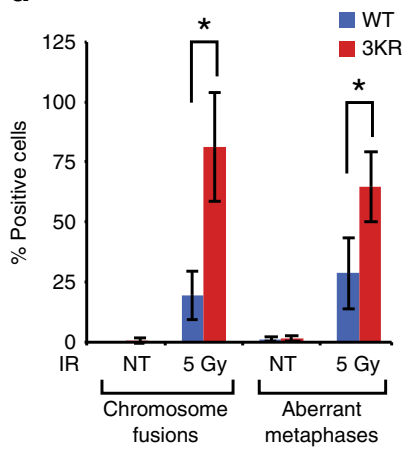

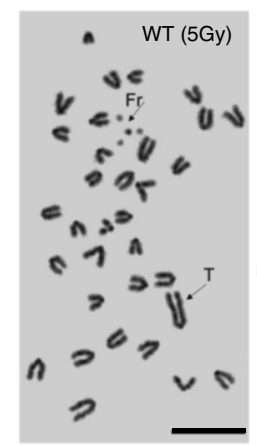

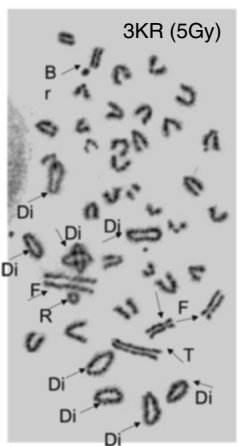

e

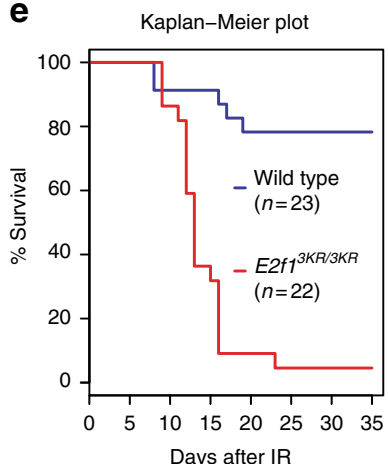

Fig. 7 The $3 K R$ mutation causes defective DNA repair phenotypes. a Primary wild-type (WT) and E2f13KR/3KR (3KR) MEFs were mock-treated (NT) or treated with $5 \mathrm{~Gy}$ of IR and allowed to recover for the indicated times. Representative confocal microscopic images show the formation and disappearance of $\gamma \mathrm{H} 2 \mathrm{AX}$ (red) and 53BP1 (green) foci and the merge with DAPI staining (blue) of nuclei. Bar, $10 \mu \mathrm{m}$. b, c Quantification of $\gamma \mathrm{H} 2 \mathrm{AX}$ foci (b) and 53BP1 foci (c) at the indicated times after IR was performed. Each treatment group was in triplicate, and in total at least 450 cells $(n>450)$ were counted per treatment group. Graphs represent average \pm SD of foci per cell. Overlaid scattered dot plot shows the distribution of foci. $P$ values were calculated by unpaired Mann-Whitney $U$ test. ${ }^{* \star \star *} P<0.0001$ is highly significant. d Primary wild-type (WT) and E2f13KR/3KR (3KR) MEFs were mock-treated (NT) or treated with $5 \mathrm{~Gy}$ of IR, and after $48 \mathrm{~h}$, metaphase spreads were prepared. Chromosome fusions including dicentrics and rings and aberrant metaphases were counted and expressed as the percentage of positive cells. Each treatment group was in triplicate $(n=3)$, and at least 50 metaphases were counted per sample in the triplicate. Graphs represent average \pm SD. $P$ values were calculated by unpaired Student's $t$ test. ${ }^{*} P \leq 0.05$ is significant. Representative metaphase spreads are shown with arrows pointing to Fr-Fragment, Di-Dicentric, R-Ring, F-Fusion, Br-Break, and T-Tandem translocation. Bar, $10 \mu \mathrm{m}$. e Wild-type $(n=23)$ and $E 2 f 13 K R / 3 K R(n=22)$ mice were exposed to $5.5 \mathrm{~Gy}$ of IR and maintained for up to 35 days. Statistical significance in survival rates between genotypes was determined using the Kaplan-Meier method and $P$ value was calculated by log-rank test. $P<0.0001$ is highly significant. Raw data of averages in graphs underlying $\mathbf{b}$-e are given in Source Data File

highlighting the role of dynamic chromatin remodeling in facilitating the efficient repair of DSBs ${ }^{49,50}$. These studies show that, immediately after a DNA break is induced, the surrounding chromatin undergoes a compaction involving the rapid recruitment of proteins, such as HP1, involved in mediating a repressive chromatin state ${ }^{51-54}$. However, this initial compact state is converted to an open, relaxed chromatin state within minutes and this requires ATM-dependent phosphorylation of downstream targets, such as KAP1 and RNF20-RNF40 $52,55-57$. This transition to an open chromatin state is also associated with Tip60-mediated $\mathrm{H} 4$ acetylation $^{33,50}$ and histone eviction at the DSB site ${ }^{58}$.

We propose that $\mathrm{p} 300 / \mathrm{CBP}$-mediated $\mathrm{H} 3$ acetylation in nucleosomes surrounding a DSB also plays an important role in this transition from a compact to a relaxed form of chromatin, perhaps upstream or co-dependent with Tip60-mediated H4 acetylation. Histone acetylation likely cooperates with nucleolin 


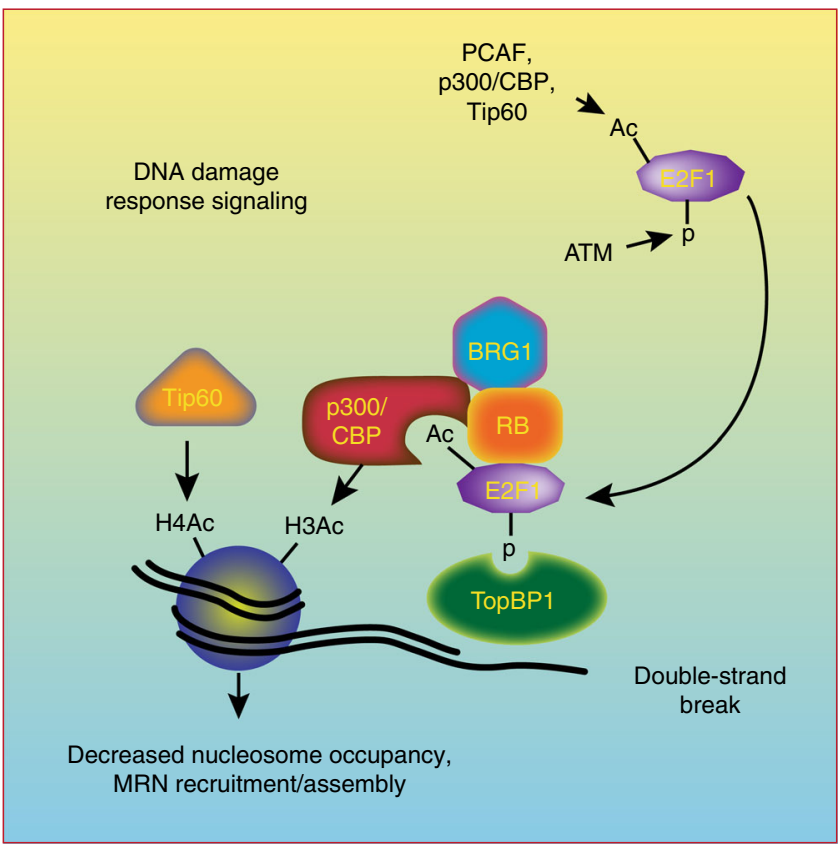

Fig. 8 Posttranslational modifications regulate E2F1 recruitment and function at DSBs. E2F1 phosphorylation recruits E2F1 and RB to sites of DNA damage via an interaction with TopBP1, while E2F1 acetylation creates a binding motif for the bromodomains of $\mathrm{p} 300$ and $\mathrm{CBP}$ allowing E2F1 and $\mathrm{RB}$ to direct p300/CBP-mediated H3 acetylation in chromatin flanking DSBs. E2F1 phosphorylation and acetylation are also important for the recruitment of Tip60 and BRG1 to sites of DNA damage and for loading of the MRN complex

and nucleosome-remodeling complexes, such as SWI/SNF, to reduce nucleosome density at the sites of DNA damage to allow efficient access to the DNA repair machinery ${ }^{19-21,58}$. Thus E2F1 and $\mathrm{RB}$ may directly participate in relaxing chromatin structure at the sites of DNA damage by recruiting both BRG1-containing SWI/SNF complexes and p300/CBP.

$\mathrm{RB}$ is known to have multiple functions that help to preserve genome integrity independent of its role in regulating gene transcription, including maintaining heterochromatin structure at repetitive sequences and mediating chromosome condensation and cohesion during mitosis ${ }^{59-62}$. We and others have also demonstrated direct roles for RB and E2F1 in DNA break repair by both $\mathrm{HR}$ and NHEJ pathways ${ }^{4,5,7}$. Interestingly, it was recently revealed that the RB and E2F1 homologs of Arabipodsis, Retinoblastoma-Related (RBR) and E2FA, both co-localize with $\gamma \mathrm{H} 2 \mathrm{AX}$ foci in response to DNA damage in an ATM- and ATRdependent manner ${ }^{63,64}$. As in humans and mice, RBR and E2FA participate in the recruitment of some DNA repair factors to the sites of damage to promote DNA repair and maintain genome integrity ${ }^{63,64}$. Whether Arabidopsis RBR and E2FA recruit histone acetyltransferases and/or nucleosome remodeling complexes to DSBs is currently unknown. Another open question is whether this direct role for $\mathrm{E} 2 \mathrm{~F} 1$ and $\mathrm{RB}$ homologs in DNA repair is conserved in other organisms.

We previously observed that NBS1 foci formation induced in response to DNA damage was significantly impaired when E2F1 was knocked out in mouse cells or knocked down in human cells ${ }^{4}$. This finding was confirmed and extended here using the IPpoI ChIP assay system, which showed impaired accumulation of NBS1 and Mre11 at DSBs in both E2f1 ${ }^{S 29 A / S 29 A}$ and E2f1 $1^{3 K R / 3 K R}$ knock-in MEFs. This indicates that both E2F1 phosphorylation and acetylation are important for the efficient loading of the MRN complex on chromatin flanking DNA break sites. The
MRN complex has multiple functions in DNA repair and DNA damage response signaling and its impaired accumulation at DSBs could explain several phenotypes observed in E2F1 mutant cells. The MRN complex is important for DNA end processing and resection to generate the single-stranded DNA required for HR repair as well as alternative NHEJ, also known as microhomology repair ${ }^{65}$. NBS1 also directly binds ATM and participates in the activation of ATM to amplify DNA damage response signaling 36,38 , consistent with the reduced ATM autophosphorylation observed in E2F1 mutant cells. Moreover, the MRN complex participates in the eviction of histones at the sites of DSBs by directly binding to and recruiting the histone chaperone nucleolin $^{58}$.

It remains unclear exactly how E2F1 knock-in mutations impair the accumulation of MRN at DSB sites. Previous studies have demonstrated impaired accumulation of MRN at the sites of DNA damage in cells depleted for Tip60, and this was associated with defective histone acetylation ${ }^{41,66}$. This suggests that E2F1dependent changes in chromatin structure may indirectly promote the loading of MRN on chromatin flanking DSBs. On the other hand, E2F1 interacts with the N-terminus of NBS1, which could indicate a direct role for E2F1 in the recruitment and/or retention of MRN at the sites of DNA damage ${ }^{67}$. These possibilities are not mutually exclusive and E2F1 may promote MRN enrichment at DSBs by both direct and indirect mechanisms. Indeed, RB is also known to physically associate with several DNA repair proteins, including components of the NHEJ repair pathway $^{5}$. These interactions, together with the E2F1-p300/CBP interaction defined here, could allow E2F1 and RB to coordinate the modification of chromatin structure with the assembly of DNA repair machinery at the sites of DSBs.

\section{Methods}

Generation of a targeted E2f1 ${ }^{3 K R}$ knock-in allele. A custom-made zinc finger nuclease (ZFN, Sigma) was designed to target mouse E2f1 sequences in exon 3 (AAACGGCGCATCTTATGACATCaccaatgtc, where the uppercase letters indicate the ZFN recognition sequence and lowercase letters represent the Folk1 cut site), approximately 70 bases 3' to the nearest codon encoding the three sites of E2F1 acetylation. A 1996 base pair donor construct was then designed (Genescript) to mutate codons 112,115 , and 120 (corresponding to human codons K117, K120, and K125 in humans) to alter protein coding from lysine to arginine (3KR mutation). One of these changes also resulted in the generation of unique $B g l I I$ recognition site, as shown in Supplementary Fig. 4a. In addition, silent point mutations were also introduced into the donor construct to destroy the ZFN recognition sequence without affecting protein sequence. The isolated donor sequence fragment and mRNAs encoding the E2f1-targeting ZFN were given to the Transgenic Animal Facility at the University of Texas MD Anderson Cancer Center, Science Park for generating founder knock-in mice (FVB strain) by pronuclear injection. Genomic DNA from the resultant pups was isolated and PCR was used to clone E2fl sequences that included the targeted codons and ZFN recognition site (forward primer: AGACATCAGACTGGGGTTGG, reverse primer: TGACAGCAAAAGCTGGAATG). Targeted alleles were identified by digestion with $B g l$ II and sequencing of E2f1 exons 2-4 was performed to confirm the presence of the targeted mutations and absence of unintended mutations. A correctly targeted founder mouse was crossed to a wild-type mouse and F1 mice positive for the $E 2 f 1^{3 K R}$ allele were further backcrossed to wild-type mice before heterozygous mice were crossed to generate homozygous $E 2 f 1^{3 K R / 3 K R}$ mice.

Animal models. Wild-type, $E 2 f 1^{S 29 A / S 29 A}$, and $E 2 f 1^{3 K R / 3 K R}$ knock-in mice are FVB strains. The wild-type FVB mice (3-4 weeks old) are purchased (Harlan Laboratories, IN) and the knock-in lines are produced in house. Generation of E2f1 ${ }^{\mathrm{S} 29 \mathrm{~A} /}$ $S 29 A$ knock-in mouse model was described earlier ${ }^{3}$ and the strain details are deposited to Mouse Genome Informatics (MGI ID: 5755411) with a symbol of

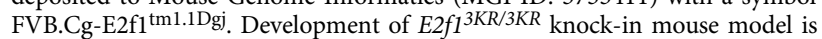

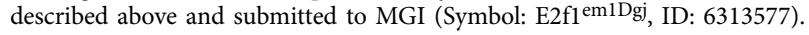

Maintenance of mice. Wild-type, $E 2 f 1^{S 29 A / S 29 A}$, and $E 2 f 1^{3 K R / 3 K R}$ knock-in mice of both sex from each strain up to age 1 year were maintained in an AAALACaccredited facility in individually ventilated cages (Micro-isolator Housing Unit; Allentown Inc, NJ) sterilized together with aspen chip bedding (Aspen Sani-chips; P.J. Murphy Forest Products Corporation, NJ). Purina Irradiated Breeder Diet (Lab Diet \#5058) was provided ad libitum and acidified reverse osmosis water was 
provided by an automated system. Cages were maintained in a room where temperature and humidity were $20-22^{\circ} \mathrm{C}$ and $55 \%$, respectively, with a 14 -h light and 10-h dark cycle and a minimum of 12 air changes per hour. Mice of both sex were randomly allocated to experiments at the age of 6-8 weeks. All animal experiments complied with the National Research Council's guide for the Care and Use of Laboratory Animals and were approved by the University of Texas MD Anderson Institutional Animal Care and Use Committee.

Cell lines and primary cell cultures. Primary MEFs of wild-type, E2f1 ${ }^{S 29 A / S 29 A}$, and $E 2 f 1^{3 K R / 3 K R}$ knock-in mice were isolated from 13.5-day-old embryos derived from crossing heterozygous or homozygous mice of each strain following standard procedures and maintained in Dulbecco's modified Eagle's medium (DMEM) supplemented with $10 \%$ heat-inactivated fetal bovine serum (FBS; Gibco), penicillin- streptomycin, and $100 \mu \mathrm{m} \beta$-mercaptoethanol at $37^{\circ} \mathrm{C}$ in $5 \% \mathrm{CO}_{2}$ and $5 \%$ $\mathrm{O}_{2}$. U2OS cells were obtained from American Type Culture Collection (ATCC, \#HTB-96) and infected with lentiviral particles expressing short hairpin RNA (shRNA) targeting $R B 1$ or $E 2 F 1^{7}$. U2OS parental and shRNA-expressing cell lines were maintained in DMEM supplemented with 10\% FBS (Sigma) and penicillin-streptomycin at $37^{\circ} \mathrm{C}$ in $5 \% \mathrm{CO}_{2}$.

Treatment conditions and reagents. IR treatment of human and mouse cell lines and of mice was performed with an RS-2000 biological irradiator (Rad Source) with the indicated doses. Where indicated, MEFs were treated with $1 \mu \mathrm{M} \mathrm{p} 300 / \mathrm{CBP}$ bromodomain-specific inhibitor I-CBP112 and $250 \mathrm{ng} / \mathrm{ml}$ of radiomimetic drug NCS. The information of reagents and resources used in this study can be found in Supplementary Table 1 or in each method's section.

Cador 5.0 protein domain microarray. CADOR 5.0 chip $^{68}$ has 174 GST fusion proteins, arrayed in duplicate on a nitrocellulose slide. The layout of the array is shown in Supplementary Fig. 1a. The middle position (M) contains GST alone as a background indicator. The list of arrayed GST fusion proteins, accession numbers, and regions cloned can be found elsewhere ${ }^{68,69}$. The complementary DNAs (cDNAs) encoding the domains listed were cloned into the pGEX-6P1 vectors by PCR using a human cDNA library (Origene, MD) and verified by DNA sequencing $^{68}$. Peptides were synthesized by W. M. Keck Biotechnology Resource Center (New Haven, CT) and CPC Scientific (Sunnyvale, CA). E2F1 peptides corresponding to human E2F1 amino acids (aa) 111-131 mono-acetylated at K117, $\mathrm{K} 120$, or K125; di-acetylated at $117 / 120,117 / 125$, and $120 / 125$; tri-acetylated at each site (3KAc); or unacetylated (UnAc) were synthesized:

E2F1 UnAc: biotin-RGRHPGKGVKSPGEKSRYETS-NH2

E2F1 3KAc: biotin-RGRHPGK ${ }^{\star} \mathrm{GVK}^{\star} \mathrm{SPGEK}^{\star}$ SRYETS-NH2

E2F1 K117Ac: biotin-RGRHPGK ${ }^{\star}$ GVKSPGEKSRYETS-NH2

E2F1 K120Ac: biotin-RGRHPGKGVK^SPGEKSRYETS-NH2

E2F1 K125Ac: biotin-RGRHPGKGVKSPGEK ${ }^{\star}$ SRYETS-NH2

E2F1 K117/120Ac: biotin-RGRHPGK ${ }^{\star} \mathrm{GVK}^{\star}$ SPGEKSRYETS-NH2

E2F1 K117/125Ac: biotin-RGRHPGK ${ }^{\star}$ GVKSPGEK ${ }^{\star}$ SRYETS-NH2

E2F1 K120/125Ac: biotin-RGRHPGKGVK ${ }^{\star}$ SPGEK ${ }^{\star}$ SRYETS-NH2

$\mathrm{K}^{*}=$ acetylated lysine

Biotinylated peptides $(10 \mu \mathrm{g})$ were pre-bound to $5 \mu \mathrm{l}$ of $\mathrm{Cy} 3-$ streptavidin or Cy5-streptavidin (Fluorolink ${ }^{\mathrm{TM}}$; Amersham Pharmacia Biotech) in $500 \mu \mathrm{l}$ of phosphate-buffered saline (PBS)-Tween 20 (PBST). The fluorescently labeled peptide was then incubated with $20 \mu \mathrm{l}$ of biotin-agarose beads to remove the free streptavidin label. Arrayed slides were blocked in PBST containing 3\% (w/v) powdered milk, followed by the addition of $400 \mu \mathrm{l}$ of fluorophore-tagged peptide. Blocking and hybridization were performed in an Atlas Glass Hybridization Chamber (Clontech). After incubation and washes, the slides were centrifuged to dry. Fluorescent signal was detected by GenePix 4200A Microarray Scanner (Molecular Devices), with the GenePix Pro Microarray Analysis Software (Molecular Devices). A $532 \mathrm{~nm}$ long pass filter was used for the detection of Cy3labeled probes and fluorescein isothiocyanate-conjugated secondary antibodies. A $635 \mathrm{~nm}$ band pass filter was used for the detection of Cy5-labeled probes. A positive signal is seen as two dots at varying angles.

Peptide pull-down assay. Biotinylated peptides $(15 \mu \mathrm{g})$ were immobilized on $25 \mu \mathrm{l}$ of streptavidin beads in $500 \mu \mathrm{l}$ of $1 \times$ mild buffer $(50 \mathrm{mM}$ Tris- $\mathrm{HCl} \mathrm{pH} 7.5,150 \mathrm{mM}$ $\mathrm{NaCl}, 5 \mathrm{mM}$ EDTA, $5 \mathrm{mM}$ EGTA, $15 \mathrm{mM} \mathrm{MgCl}_{2}$, and $0.1 \% \mathrm{NP}-40$ ), then incubated with $2-3 \mu \mathrm{g}$ of GST fusion protein for $1 \mathrm{~h}$. After washing, the beads were boiled in Laemmli buffer, separated by $10 \%$ sodium dodecyl sulfate

(SDS)-polyacrylamide gel electrophoresis (PAGE) and subjected to western blot analysis using an anti-GST antibody.

Purification of GST fusion proteins. For NMR titration assays, GST fusion p300BD (1040-1161 aa) and His-6x fusion p300-BRP (1051-1278 aa) proteins were expressed in minimal media supplemented with $50-150 \mu \mathrm{M} \mathrm{ZnCl}{ }_{2}$ and ${ }^{15} \mathrm{NH}_{4} \mathrm{Cl}$. After induction with $0.4-1.0 \mathrm{mM}$ isopropyl $\beta$-D-1-thiogalactopyranoside (IPTG) for $18 \mathrm{~h}$ at $16^{\circ} \mathrm{C}$, bacteria were harvested through centrifugation and lysed by sonication. GST fusion proteins were purified on glutathione-Agarose $4 \mathrm{~B}$ beads (Thermo Fisher Sci) and the GST tag was cleaved with PreScission protease. Histagged proteins were purified on Ni-NTA agarose beads and washed and eluted with imidazole. The His-tag was removed by cleavage with PreScission protease. Proteins were concentrated using Millipore concentrators (Millipore).

For GST pull-down and peptide pull-down assays, indicated GST-fusion proteins were overexpressed in Escherichia coli DH5a cells (Life Technologies) by induction with a final concentration of $0.1 \mathrm{mM}$ IPTG at $30^{\circ} \mathrm{C}$ for $4 \mathrm{~h}$. Cells were pelleted and resupended in PBS, then subjected to sonication. The resulting lysates were centrifuged at maximum speed for $10 \mathrm{~min}$, and the GST fusion proteins were then batch-purified from extracts by binding to glutathione-Sepharose $4 \mathrm{~B}$ beads and washed in PBS according to the manufacturer's instructions. The purified proteins were eluted from the beads with elution buffer $(30 \mathrm{mM}$ Glutathione reduced, $100 \mathrm{mM}$ Tris- $\mathrm{HCl}, \mathrm{pH} 8.0$, and $120 \mathrm{mM} \mathrm{NaCl}$ ). The purified proteins were stored in the elution buffer at $-80^{\circ} \mathrm{C}$.

NMR experiments. NMR experiments were performed at $298 \mathrm{~K}$ on a Varian INOVA $600 \mathrm{MHz}$ spectrometer. ${ }^{1} \mathrm{H},{ }^{15} \mathrm{~N}$ HSQC spectra of uniformly ${ }^{15} \mathrm{~N}$-labeled p300 BD or BRP $(0.1-0.15 \mathrm{mM})$ in buffer (BD: $25 \mathrm{mM}$ Tris at $\mathrm{pH} 6.8,150 \mathrm{mM}$ $\mathrm{NaCl}, 2 \mathrm{mM}$ dithiothreitol or BRP: $10 \mathrm{mM}$ Hepes, $150 \mathrm{mM} \mathrm{NaCl}, 2 \%$ glycerol) and $\sim 8 \% \mathrm{D}_{2} \mathrm{O}$ were collected as E2F1 peptides were added stepwise into the protein samples. NMR data were processed and analyzed with NMRPipe and NMRDraw ${ }^{70}$ NMRPipe is from NIST IBBR and available at https://www.ibbr.umd.edu/nmrpipe/ index.html

GST pull-down and IP blotting. For GST pull-down experiments, cells were treated as indicated and harvested in cold PBS followed by resuspension in cell lysis buffer $(20 \mathrm{mM}$ Tris at $\mathrm{pH} 7.5,150 \mathrm{mM} \mathrm{NaCl}, 1 \%$ Triton X-100, $1 \mathrm{mM}$ EDTA, $1 \mathrm{mM}$ EGTA, $2.5 \mathrm{mM}$ Sodium pyrophosphate, protease inhibitor cocktail, phosphatase inhibitor, and HDAC inhibitors - 300 nM TSA, $2 \mu \mathrm{M}$ EX-527). After aliquoting $5 \%$ of the lysates as input, 1-2 $\mathrm{mg}$ of lysates were pre-cleared by incubating with $25 \mu \mathrm{g}$ of purified GST for $2 \mathrm{~h}$ at $4{ }^{\circ} \mathrm{C}$ and pulled down using glutathioneSepharose $4 \mathrm{~B}$ beads as per the manufacturer's directions. Pre-cleared lysates were divided equally to incubate with $15 \mu \mathrm{g}$ of purified GST or GST-TopBP1 overnight at $4{ }^{\circ} \mathrm{C}$. Next day, pull-down was performed using glutathione beads. Both samples and input were mixed with Laemmli buffer, boiled for $5 \mathrm{~min}$, separated by SDSPAGE, and subjected to western blot analysis ${ }^{7}$. See Supplementary Table 2 for the list of antibodies used.

For co-immunoprecipitation, cell lysates were prepared as mentioned above. Four hundred $\mu \mathrm{g}$ of lysates were pre-cleared, followed by IP with IgG control or $2.5 \mu \mathrm{g}$ of antibody to E2F1 (C-20) and pulled down by protein $\mathrm{G}$ magnetic beads (Cell Signaling Technology). Immunocomplexes were eluted by boiling the beads with Laemmli buffer, followed by western blotting with acetylated lysine or E2F1. For western blot analysis of cell lysates, 15-100 $\mu \mathrm{g}$ of samples were mixed with Laemmli buffer, boiled for $5 \mathrm{~min}$, run on SDS-PAGE, and then transferred onto a polyvinylidene difluoride membrane (Amersham Hybond). Blots were blocked in PBS-Tween 20 (PBST) or TBST containing 5\% non-fat dry milk or bovine serum albumin respectively, and then incubated with primary antibody in the blocking buffer overnight at $4{ }^{\circ} \mathrm{C}$. The list of antibodies used in this study is given in Supplementary Table 2 . After washing and incubating with horseradish peroxidase (HRP)-conjugated secondary antibodies (Santa Cruz Biotechnology and Abcam), the membrane was subjected to ECL (Enhanced Chemiluminescence) detection as per the supplier's instructions. Signal was exposed to Autorad film (GeneMate) and developed using Medical Film processor (Konica Minolta SRX-101A).

ChIP and qPCR. Transduction of MEFs and U2OS cells with retrovirus expressing HA-ER ${ }^{*}$-I-PpoI enzyme was performed twice to increase infection efficiency and treated with $2 \mu \mathrm{M}$ 4-hydroxy tamoxifen (Sigma) for $12 \mathrm{~h}$ to induce DNA damage ${ }^{22}$ Cells were crosslinked by adding formaldehyde ( $1 \%$ final concentration) followed by quenching with glycine (Sigma) at $1.25 \mathrm{mM}$ final concentration, then harvested. Cell pellets were resuspended in SDS lysis buffer (1\% SDS, $10 \mathrm{mM}$ EDTA, $50 \mathrm{mM}$ Tris at $\mathrm{pH}$ 8.1, protease inhibitor cocktail, phosphatase inhibitor, and HDAC inhibitors - $300 \mathrm{nM}$ TSA, $2 \mu \mathrm{M}$ EX-527) and the lysates were sonicated. After diluting with ChIP dilution buffer (0.01\% SDS, $1.2 \mathrm{mM}$ EDTA, $16.7 \mathrm{mM}$ Tris at $\mathrm{pH}$ $8.1,1.1 \%$ Triton X-100, $167 \mathrm{mM} \mathrm{NaCl}$, protease inhibitor cocktail, phosphatase inhibitor, and HDAC inhibitors - $300 \mathrm{nM}$ TSA, $2 \mu \mathrm{M}$ EX-527), the lysates were pre-cleared and subjected to IP with the antibodies indicated (Supplementary Table 2). In all, $10 \%$ input was aliquoted separately. Next day, the DNA-bound immunocomplexes were pulled down by ChIP-Grade Protein G Magnetic beads and subjected to serial washes with buffers of low salt, high salt, $\mathrm{LiCl}$, and TE. Then the crosslinked DNA was eluted with Elution buffer (1\% SDS and $0.1 \mathrm{M} \mathrm{NaHCO}_{3}$ ) at $65^{\circ} \mathrm{C}$. Subsequently, the samples including input were incubated with $5 \mathrm{M} \mathrm{NaCl}$ at $65^{\circ} \mathrm{C}$ overnight for reverse crosslinking. After RNase A (Sigma) and Proteinase $\mathrm{K}$ (Sigma) treatment, the DNA was eluted using the QIAquick PCR Purification Kit (Qiagen) as per the manufacturer's instructions.

Occupancy of the DNA-bound proteins was measured by subjecting eluted DNA to qPCR using 7500 Fast Real-Time PCR system (Applied Biosystems) with primers for the indicated loci. Primers are listed in Supplementary Table 3. The percentage of input was calculated by dividing the amount of DNA obtained from the IP of the given factor by the total amount of DNA (input) and normalized for background signal (non-specific IgG control). Each experiment was carried out in triplicate and the results were expressed as relative enrichment, which represents 
the ratio between the normalized percentage of input of infected cells and uninfected cells.

IR treatment of mice and survival study post-IR. Pups of wild-type and $E 2 f 1^{3 K R /}$ $3 K R$ strain were weaned at 21 days according to sex, then aged to 6-8 weeks prior to IR treatment. Mice were shifted to IR treatment room and transferred into sterile treatment cages in the biosafety cabinet. After $5.5 \mathrm{~Gy}$ of irradiation (up to 5 mice per treatment cage), they were returned to biosafety hood and transferred into sterile cages (with sterile feed, bedding, and water containing $50 \mathrm{mg} / \mathrm{ml}$ of Clavamox). In the biosafety hood, antibiotic water bottles were changed out every 5 days and cages were changed out every 7 days for animals remaining housed after treatment. Mice were group housed if possible; single housed if need to prevent fighting. Mice were monitored daily for symptoms of ill-health and were euthanized if they became moribund as per federal and institutional guidelines.

Cleaved Lamin A staining and immunohistochemistry (IHC). Wild-type and $E 2 f 1^{3 K R / 3 K R}$ mice were subjected to IR treatment as described above and sacrificed $2 \mathrm{~h}$ later. Thymus and spleen tissues were collected, mounted on to cassettes, fixed in $10 \%$ neutral buffered formalin for $24-48 \mathrm{~h}$, and then moved to $70 \%$ ethanol and paraffin embedded. Tissue sections were deparaffinized in xylene or xylene substitute followed by graded alcohols $(100 \%, 95 \%$ Ethanol) to water. Endogenous peroxidase activity was blocked with $3 \% \mathrm{H}_{2} \mathrm{O}_{2}$ for $10 \mathrm{~min}$ in water. Antigen was retrieved with $10 \mathrm{mM}$ Citrate Buffer at $\mathrm{pH} 6.0$ in a microwave oven for 3 min at $100 \%$ power followed by $15 \mathrm{~min}$ at $50 \%$ power, then cooled down for $20 \mathrm{~min}$ Binding of non-specific antibody was blocked by incubating the slides with blocking reagent (Biocare Medical) for $10 \mathrm{~min}$. Then the slides were incubated with primary cleaved Lamin A antibody overnight at $4{ }^{\circ} \mathrm{C}$ (refer Supplementary Table 2 for antibody information). Next, these were washed twice and incubated with Envision plus labeled polymer and anti-rabbit-HRP (Dako) for $30 \mathrm{~min}$ at room temperature. After washing twice, these were incubated with DAB (Dako) for monitoring staining development. Finally, these were counterstained and dehydrated, and coverslips were placed on the slides for viewing.

A set of 48 glass slides stained for IHC Lamin A were scanned by using the Aperio ScanScope imaging platform (Leica Biosystems, IL) with a $\times 20$ objective at a spatial sampling period of $0.47 \mu \mathrm{m} /$ pixel. After saving each digital image, a Genie classifier algorithm was trained to quantitate cleaved Lamin A-positive and -negative nuclei. Whole-slide images were viewed and analyzed by using desktop personal computers equipped with the free ScanScope software.

RNA isolation and qPCR of gene expression. Total RNA was isolated from wildtype and knock-in MEFs before and $3 \mathrm{~h}$ after treatment with NCS using the GenElute mammalian Total RNA Miniprep Kit (Sigma) following the supplier's instructions. For tissue samples, wild-type and $E 2 f 1^{3 K R / 3 K R}$ mice were subjected to $5.5 \mathrm{~Gy}$ of IR treatment and sacrificed $2 \mathrm{~h}$ later. Thymus, spleen, and liver tissues were collected and subjected to total RNA isolation using the same kit as per the instructions. On-column DNase I digestion was performed for all samples before eluting the RNA. Five hundred ng of total RNA was used to prepare cDNA using SuperScript II Reverse transcriptase with random hexamer primer. cDNAs were subjected to real-time qPCR with gene-specific primers. Primers are listed in Supplementary Table 3. Relative mRNA quantification was performed by comparative $C_{\mathrm{T}}$ method using Gapdh as an internal control.

RNA-seq and GSEA. Total RNA was isolated from wild-type and $E 2 f 1^{3 K R / 3 K R}$ MEFs before and $3 \mathrm{~h}$ after treatment with NCS using the GenElute mammalian Total RNA Miniprep Kit (Sigma) following the supplier's instructions. DNase Itreated RNA samples were fragmented and tagged at both ends for stranded library preparation using the TrueSeq Stranded mRNA Kit (Illumina). The mRNA-seq run was performed on the HiSeq 3000 platform (Illumina). Approximately 40-60 million reads were acquired per sample. The differential expression analysis for mRNA-seq data was performed with DESeq2 bioconductor R package with the cutoff of False Discovery Rate (FDR) $q \leq 0.05$. The normalized read count was generated from built-in function in DESeq2.

Sequenced tags were analyzed by GSEA downloaded from Broad Institute at http://software.broadinstitute.org/gsea/index.jsp. The mouse gene sets for Gene Ontology (GO) was prebuilt and can be downloaded from http://www.bioinformatics. org/go2msig/. The high-quality GO annotations for biological process GO terms with MsigDB format (.gmt) were utilized. Additional two input files, expression dataset file (.txt) and phenotype labels file (.cls), were generated according to the file formats described in GSEA user guide and GSEA data format guide.

IF staining and confocal microscopy. Wild-type and $E 2 f 1^{3 K R / 3 K R}$ MEFs $\left(0.5 \times 10^{6}\right.$ cells) were seeded on $35 \mathrm{~mm}$ FluoroDish with $0.17 \mathrm{~mm}$ cover glass bottom (World Precision Instruments), $24 \mathrm{~h}$ prior to experiment. For $\gamma \mathrm{H} 2 \mathrm{AX}$ and 53BP1 foci formation and clearance assay, cells were treated with IR and, at the indicated times, rinsed with cold PBS, and fixed for $15 \mathrm{~min}$ at room temperature in $4 \%$ paraformaldehyde and $1 \%$ sucrose. For CBP and p300 IF experiments, cells were irradiated, harvested $2 \mathrm{~h}$ post-IR, and subjected to in situ extraction protocol as follows. After rinsing with cold PBS, cells were incubated $5 \mathrm{~min}$ on ice with pre- extraction buffer (25 mM HEPES pH 7.5, $50 \mathrm{mM} \mathrm{NaCl}, 1 \mathrm{mM}$ EDTA, $3 \mathrm{mM}$ $\mathrm{MgCl}_{2}, 300 \mathrm{mM}$ Sucrose, $0.5 \%$ Triton X-100), followed by 5 -min incubation on ice with stripping buffer (10 mM Tris pH 7.4,10 mM NaCl, $3 \mathrm{mM} \mathrm{MgCl}_{2}, 1 \mathrm{mM}$ EDTA, 1\% Tween-20, 0.5\% Na-Deoxycholate), and fixed as above.

After washing, cells were permeabilized with $0.5 \%$ Triton X-100 and blocked in Background Sniper for $15 \mathrm{~min}$. Antibodies against CBP, p300, $\gamma \mathrm{H} 2 \mathrm{AX}$, and 53BP1 were diluted with DaVinci Green antibody diluent and incubated with the cells overnight at $4{ }^{\circ} \mathrm{C}$ (See Supplementary Table 2 for antibody information). After washing, cells were incubated with appropriate fluorophore-conjugated secondary antibodies (Thermo Fisher Sci), stained with 4,6-diamidino-2-phenylindole and mounted in SlowFade Diamond Anti-fade mountant (Thermo Fisher Sci). Laser scanning confocal microscopy was performed using a Zeiss LSM880 and $\times 63$ oil (1.4 NA) Plan/Apo objective with a pinhole aperture of 1-1.5 AU. Foci quantification of $5 \times 5$ tile scans were performed using the Imaris (Bitplane, v9.0) image analysis software. Cells module and custom algorithms specific to CBP, p300, $\gamma \mathrm{H} 2 \mathrm{AX}$ and $53 \mathrm{BP} 1$ foci. Each treatment group was in triplicate, and in total at least 450 cells were counted per treatment group. Images were captured and processed using identical microscope settings and foci parameters.

Metaphase chromosome spread preparation. Wild-type and $E 2 f 1^{3 K R / 3 K R} \mathrm{MEFs}$ were irradiated as indicated and, $38 \mathrm{~h}$ after treatment, incubated with $0.1 \mu \mathrm{g} / \mathrm{ml}$ colcemid for $10 \mathrm{~h}$. Cells were trypsinized, pelleted down, kept in a hypotonic solution $(0.075 \mathrm{M} \mathrm{KCl})$ for $15 \mathrm{~min}$ at room temperature, then fixed with 1:3 acetic acid:methanol, and spread on a slide. Metaphase chromosome aberrations of breaks, dicentrics, rings, chromosome fusions, fragments, and other aberrations were analyzed by Nikon Microscope with $\times 63$ oil immersion objective. Images were taken using Imaging system from Applied Spectral Imaging (Carlsbad, CA). Approximately, 150 metaphases were counted per treatment. Each treatment group was set up in triplicate, and at least 50 metaphases were counted per sample in the triplicate.

Statistical information. For statistical analysis of mice survival curve, a Kaplan-Meier estimate was generated and analyzed for statistical significance with log-rank test. The difference in the survival rates between genotypes is highly significant with values of $P<0.0001$. All other quantitative experiments were carried out in triplicate, and graphs represent average \pm standard deviation (SD). Statistical analysis was performed using unpaired Student's $t$ test for all experiments except foci quantification by IF staining in which unpaired Mann-Whitney $U$ test was used. Significance was determined, with $P \leq 0.05\left(^{*}\right)$ considered to be significant and $P \leq 0.01\left(^{(*)}\right.$ and $P<0.0001\left(^{* * * *}\right.$ ) are considered to be highly significant. For GSEA of RNA-seq data, significantly enriched pathways between genotypes were determined with cutoff of FDR value, $q \leq 0.05$.

Reporting summary. Further information on research design is available in the Nature Research Reporting Summary linked to this article.

\section{Data availability}

Knock-in mouse models used in this study are registered in Mouse Genome Informatics (MGI) database. Strain details of $E 2 \mathrm{fl}^{\mathrm{S} 29 \mathrm{~A} / \mathrm{S} 29 \mathrm{~A}}$ (FVB.Cg-E2f1 ${ }^{\text {tml.1Dgi) }}$ can be found in MGI ID:5755411 [http://www.informatics.jax.org/allele/MGI:5637520] and E2f13 3 KR/3KR (E2f1 ${ }^{\text {emlDgj) }}$ in MGI ID:6313577 [http://www.informatics.jax.org/allele/key/882523]. RNA-seq data are available in Gene Expression Omnibus (GEO) repository under accession GSE135360. All data supporting the findings of this study are available within the article and its supplementary information files. Additional information and relevant data will be available from the corresponding author upon reasonable request. Supplementary Data 5 contains raw data of blots/gels underlying Fig. 1b, c; $2 a$, b; $4 b, c$; 5b; 6b, d; and Supplementary Figs. 1c; 2a; 3d; 4b; 6e; 7a, b. Source Data file contains raw data of all reported averages in graphs and charts underlying main figures, Fig. 3a-c; Fig. 4a, d and e; Fig. 5a, d-f; Fig. 6a and c; Fig. 7b-e; and Supplementary Figs. 2b, c; 3b, c and e; $4 c ; 5 a-c$ and $6 a-d$.

\section{Code availability}

Data in this manuscript are generated using commonly available commercial software and algorithms and are detailed in the corresponding "Methods" section. Specific computer code is not applicable.

Received: 4 April 2019; Accepted: 3 October 2019; Published online: 30 October 2019

\section{References}

1. DeGregori, J. \& Johnson, D. G. Distinct and overlapping roles for E2F family members in transcription, proliferation and apoptosis. Curr. Mol. Med. 6, 739-748 (2006). 
2. Johnson, D. G. \& Degregori, J. Putting the oncogenic and tumor suppressive activities of E2F into context. Curr. Mol. Med. 6, 731-738 (2006).

3. Biswas, A. K., Mitchell, D. L. \& Johnson, D. G. E2F1 responds to ultraviolet radiation by directly stimulating DNA repair and suppressing carcinogenesis. Cancer Res. 74, 3369-3377 (2014).

4. Chen, J. et al. E2F1 promotes the recruitment of DNA repair factors to sites of DNA double-strand breaks. Cell Cycle 10, 1287-1294 (2011).

5. Cook, R. et al. Direct involvement of retinoblastoma family proteins in DNA repair by non-homologous end-joining. Cell Rep. 10, 2006-2018 (2015)

6. Guo, R. et al. E2F1 localizes to sites of UV-induced DNA damage to enhance nucleotide excision repair. J. Biol. Chem. 285, 19308-19315 (2010).

7. Velez-Cruz, R. et al. RB localizes to DNA double-strand breaks and promotes DNA end resection and homologous recombination through the recruitment of BRG1. Genes Dev. 30, 2500-2512 (2016).

8. Liu, K., Lin, F. T., Ruppert, J. M. \& Lin, W. C. Regulation of E2F1 by BRCT domain-containing protein TopBP1. Mol. Cell. Biol. 23, 3287-3304 (2003).

9. Lin, W. C., Lin, F. T. \& Nevins, J. R. Selective induction of E2F1 in response to DNA damage, mediated by ATM- dependent phosphorylation. Genes Dev. 15, 1833-1844 (2001).

10. Ianari, A., Gallo, R., Palma, M., Alesse, E. \& Gulino, A. Specific role for PCAF acetylytransferase activity in E2F1 stabilization in response to DNA damage. J. Biol. Chem. 279, 20830-30835 (2004).

11. Martinez-Balbas, M. A., Bauer, U. M., Nielsen, S. J., Brehm, A. \& Kouzarides, T. Regulation of E2F1 activity by acetylation. EMBO J. 19, 662-671 (2000).

12. Galbiati, L., Mendoza-Maldonado, R., Gutierrez, M. I. \& Giacca, M. Regulation of E2F-1 after DNA damage by p300-mediated acetylation and ubiquitination. Cell Cycle 4, 930-939 (2005).

13. Marzio, G. et al. E2F family members are differentially regulated by reversible acetylation. J. Biol. Chem. 275, 10887-10892 (2000).

14. Van Den Broeck, A., Nissou, D., Brambilla, E., Eymin, B. \& Gazzeri, S. Activation of a Tip60/E2F1/ERCC1 network in human lung adenocarcinoma cells exposed to cisplatin. Carcinogenesis 33, 320-325 (2012).

15. Wang, C. et al. Interactions between E2F1 and SirT1 regulate apoptotic response to DNA damage. Nat. Cell Biol. 8, 1025-1031 (2006).

16. Pediconi, N. et al. Differential regulation of E2F1 apoptotic target genes in response to DNA damage. Nat. Cell Biol. 5, 552-558 (2003).

17. Ferri, E., Petosa, C. \& McKenna, C. E. Bromodomains: structure, function and pharmacology of inhibition. Biochem. Pharmacol. 106, 1-18 (2015).

18. Chiu, L. Y., Gong, F. \& Miller, K. M. Bromodomain proteins: repairing DNA damage within chromatin. Philos. Trans. R. Soc. Lond. B Biol. Sci. 372, 20160286 (2017).

19. Lee, H. S., Park, J. H., Kim, S. J., Kwon, S. J. \& Kwon, J. A cooperative activation loop among SWI/SNF, gamma-H2AX and H3 acetylation for DNA double-strand break repair. EMBO J. 29, 1434-1445 (2010).

20. Ogiwara, H. et al. Histone acetylation by CBP and p300 at double-strand break sites facilitates SWI/SNF chromatin remodeling and the recruitment of non-homologous end joining factors. Oncogene 30, 2135-2146 (2011).

21. Qi, W. et al. Acetyltransferase p300 collaborates with chromodomain helicase DNA-binding protein 4 (CHD4) to facilitate DNA double-strand break repair. Mutagenesis 31, 193-203 (2016).

22. Berkovich, E., Monnat, R. J. Jr. \& Kastan, M. B. Assessment of protein dynamics and DNA repair following generation of DNA double-strand breaks at defined genomic sites. Nat. Protoc. 3, 915-922 (2008).

23. Das, C., Lucia, M. S., Hansen, K. C. \& Tyler, J. K. CBP/p300-mediated acetylation of histone $\mathrm{H} 3$ on lysine 56. Nature 459, 113-117 (2009).

24. Jin, Q. et al. Distinct roles of GCN5/PCAF-mediated H3K9ac and CBP/p300mediated $\mathrm{H} 3 \mathrm{~K} 18 / 27 \mathrm{ac}$ in nuclear receptor transactivation. EMBO J. 30, 249-262 (2011).

25. Ogiwara, H. \& Kohno, T. CBP and p300 histone acetyltransferases contribute to homologous recombination by transcriptionally activating the BRCA1 and RAD51 genes. PLoS ONE 7, e52810 (2012).

26. Schiltz, R. L. et al. Overlapping but distinct patterns of histone acetylation by the human coactivators $\mathrm{p} 300$ and PCAF within nucleosomal substrates. J. Biol. Chem. 274, 1189-1192 (1999).

27. Vempati, R. K. et al. p300-mediated acetylation of histone H3 lysine 56 functions in DNA damage response in mammals. J. Biol. Chem. 285, 28553-28564 (2010).

28. Pankotai, T., Bonhomme, C., Chen, D. \& Soutoglou, E. DNAPKcs-dependent arrest of RNA polymerase II transcription in the presence of DNA breaks. Nat. Struct. Mol. Biol. 19, 276-282 (2012).

29. Picaud, S. et al. Generation of a selective small molecule inhibitor of the CBP/ p300 bromodomain for leukemia therapy. Cancer Res. 75, 5106-5119 (2015).

30. Moroni, M. C. et al. Apaf-1 is a transcriptional target for E2F and p53. Nat. Cell Biol. 3, 552-558 (2001).

31. Nahle, Z. et al. Direct coupling of the cell cycle and cell death machinery by E2F. Nat. Cell Biol. 4, 859-864 (2002).

32. Murr, R. et al. Histone acetylation by Trrap-Tip60 modulates loading of repair proteins and repair of DNA double-strand breaks. Nat. Cell Biol. 8, 91-99 (2006).
33. Sun, $\mathrm{Y}$. et al. Histone $\mathrm{H} 3$ methylation links DNA damage detection to activation of the tumour suppressor Tip60. Nat. Cell Biol. 11, 1376-1382 (2009).

34. $\mathrm{Xu}, \mathrm{Y}$. et al. The $\mathrm{p} 400$ ATPase regulates nucleosome stability and chromatin ubiquitination during DNA repair. J. Cell. Biol. 191, 31-43 (2010).

35. Sobczak, M., Pitt, A. R., Spickett, C. M., Robaszkiewicz, A. PARP1 co-regulates EP300-BRG1-dependent transcription of genes involved in breast cancer cell proliferation and DNA repair. Cancers 11, E1539 (2019).

36. Lee, J. H. \& Paull, T. T. ATM activation by DNA double-strand breaks through the Mre11-Rad50-Nbs1 complex. Science 308, 551-554 (2005).

37. Lee, J. H. et al. Distinct functions of Nijmegen breakage syndrome in ataxia telangiectasia mutated-dependent responses to DNA damage. Mol. Cancer Res. 1, 674-681 (2003).

38. Uziel, T. et al. Requirement of the MRN complex for ATM activation by DNA damage. EMBO J. 22, 5612-5621 (2003).

39. Bakkenist, C. J. \& Kastan, M. B. DNA damage activates ATM through intermolecular autophosphorylation and dimer dissociation. Nature 421, 499-506 (2003).

40. Nakad, R. \& Schumacher, B. DNA damage response and immune defense: links and mechanisms. Front. Genet. 7, 147 (2016).

41. Chailleux, C. et al. Physical interaction between the histone acetyl transferase Tip60 and the DNA double-strand breaks sensor MRN complex. Biochem. J. 426, 365-371 (2010).

42. Fry, C. J. et al. Activation of the murine dihydrofolate reductase promoter by E2F1. A requirement for CBP recruitment. J. Biol. Chem. 274, 15883-15891 (1999).

43. Lang, S. E., McMahon, S. B., Cole, M. D. \& Hearing, P. E2F transcriptional activation requires TRRAP and GCN5 cofactors. J. Biol. Chem. 276 32627-32634 (2001).

44. Swarnalatha, M., Singh, A. K. \& Kumar, V. Promoter occupancy of MLL1 histone methyltransferase seems to specify the proliferative and apoptotic functions of E2F1 in a tumour microenvironment. J. Cell. Sci. 126, 4636-4646 (2013).

45. Taubert, S. et al. E2F-dependent histone acetylation and recruitment of the Tip60 acetyltransferase complex to chromatin in late G1. Mol. Cell. Biol. 24, 4546-4556 (2004)

46. Smerdon, M. J., Lan, S. Y., Calza, R. E. \& Reeves, R. Sodium butyrate stimulates DNA repair in UV-irradiated normal and Xeroderma pigmentosum human fibroblasts. J. Biol. Chem. 257, 13441-13447 (1982).

47. Smerdon, M. J. \& Lieberman, M. W. Nucleosome rearrangement in human chromatin during UV-induced DNA- repair synthesis. Proc. Natl Acad. Sci. USA 75, 4238-4241 (1978).

48. Guo, R., Chen, J., Mitchell, D. L. \& Johnson, D. G. GCN5 and E2F1 stimulate nucleotide excision repair by promoting $\mathrm{H} 3 \mathrm{~K} 9$ acetylation at sites of damage. Nucleic Acids Res. 39, 1390-1397 (2011)

49. Bakkenist, C. J. \& Kastan, M. B. Chromatin perturbations during the DNA damage response in higher eukaryotes. DNA Repair (Amst.) 36, 8-12 (2015).

50. Gursoy-Yuzugullu, O., House, N. \& Price, B. D. Patching broken DNA: nucleosome dynamics and the repair of DNA breaks. J. Mol. Biol. 428, 1846-1860 (2016)

51. Ayoub, N., Jeyasekharan, A. D., Bernal, J. A. \& Venkitaraman, A. R. HP1-beta mobilization promotes chromatin changes that initiate the DNA damage response. Nature 453, 682-686 (2008).

52. Ayrapetov, M. K., Gursoy-Yuzugullu, O., Xu, C., Xu, Y. \& Price, B. D. DNA double-strand breaks promote methylation of histone $\mathrm{H} 3$ on lysine 9 and transient formation of repressive chromatin. Proc. Natl Acad. Sci. USA 111, 9169-9174 (2014)

53. Baldeyron, C., Soria, G., Roche, D., Cook, A. J. \& Almouzni, G. HP1alpha recruitment to DNA damage by p150CAF-1 promotes homologous recombination repair. J. Cell. Biol. 193, 81-95 (2011).

54. Chou, D. M. et al. A chromatin localization screen reveals poly (ADP ribose) regulated recruitment of the repressive polycomb and NuRD complexes to sites of DNA damage. Proc. Natl Acad. Sci. USA 107, 18475-18480 (2010).

55. Goodarzi, A. A. et al. ATM signaling facilitates repair of DNA double-strand breaks associated with heterochromatin. Mol. Cell 31, 167-177 (2008).

56. Moyal, L. et al. Requirement of ATM-dependent monoubiquitylation of histone H2B for timely repair of DNA double-strand breaks. Mol. Cell 41, 529-542 (2011).

57. Ziv, Y. et al. Chromatin relaxation in response to DNA double-strand breaks is modulated by a novel ATM- and KAP-1 dependent pathway. Nat. Cell Biol. 8, 870-876 (2006).

58. Goldstein, M., Derheimer, F. A., Tait-Mulder, J. \& Kastan, M. B. Nucleolin mediates nucleosome disruption critical for DNA double-strand break repair. Proc. Natl Acad. Sci. USA 110, 16874-16879 (2013).

59. Coschi, C. H. et al. Haploinsufficiency of an RB-E2F1-Condensin II complex leads to aberrant replication and aneuploidy. Cancer Discov. 4, 840-853 (2014). 
60. Coschi, C. H. et al. Mitotic chromosome condensation mediated by the retinoblastoma protein is tumor-suppressive. Genes Dev. 24, 1351-1363 (2010).

61. Ishak, C. A. et al. An RB-EZH2 complex mediates silencing of repetitive DNA sequences. Mol. Cell 64, 1074-1087 (2016).

62. Velez-Cruz, R. \& Johnson, D. G. The retinoblastoma (RB) tumor suppressor: pushing back against genome instability on multiple fronts. Int. J. Mol. Sci. 18, E1776 (2017).

63. Biedermann, S. et al. The retinoblastoma homolog RBR1 mediates localization of the repair protein RAD51 to DNA lesions in Arabidopsis. EMBO J. 36, 1279-1297 (2017).

64. Horvath, B. M. et al. Arabidopsis RETINOBLASTOMA RELATED directly regulates DNA damage responses through functions beyond cell cycle control. EMBO J. 36, 1261-1278 (2017).

65. Xie, A., Kwok, A. \& Scully, R. Role of mammalian Mre11 in classical and alternative nonhomologous end joining. Nat. Struct. Mol. Biol. 16, 814-818 (2009).

66. Ikura, M. et al. Acetylation of histone H2AX at Lys 5 by the TIP60 histone acetyltransferase complex is essential for the dynamic binding of NBS1 to damaged chromatin. Mol. Cell. Biol. 35, 4147-4157 (2015).

67. Maser, R. S. et al. Mre11 complex and DNA replication: linkage to E2F and sites of DNA synthesis. Mol. Cell. Biol. 21, 6006-6016 (2001).

68. Kim, J. et al. Tudor, MBT and chromo domains gauge the degree of lysine methylation. EMBO Rep. 7, 397-403 (2006).

69. Yang, Y. et al. TDRD3 is an effector molecule for arginine-methylated histone marks. Mol. Cell 40, 1016-1023 (2010).

70. Klein, B. J. et al. The histone-H3K4-specific demethylase KDM5B binds to its substrate and product through distinct PHD fingers. Cell Rep. 6, 325-335 (2014).

\section{Acknowledgements}

We thank B. Brooks and R. Deen for manuscript preparation; J. Holcomb for graphics; J. Orona and M. Portis for expert technical assistance; Ray. S for preliminary study; Lin. K for biostatistics; C. Sagum and the Protein Array and Analysis Core (supported by CPRIT RP180804) and J. Terpstra and C. Jeter of the Flow Cytometry \& Cellular Imaging Core (supported by CPRIT RP170628) for assistance with IF; JJ Shen and the NextGeneration Sequencing Core (supported by CPRIT RP120348 and RP170002) for RNASeq; A. Multani and the Molecular Cytogenetics Facility for metaphase spreads analysis; D. Hollowell and the Transgenic Animal Core for generating knock-in mice; C. Perez of the Research Histology, Pathology and Imaging services for IHC; and Dale Weiss and colleagues in the Research Animal Support Facility for animal care. This work was supported by grants from the Cancer Prevention and Research Institute of Texas (RP140222 to D.G.J.); the National Institutes of Health (CA214723 to D.G.J., GM100907 to T.G.K. and Cancer Core Support Grant CA016672); and institutional funding from the Department of Epigenetics and Molecular Carcinogenesis, the Center for Cancer Epigenetics, and the Center for Genetics and Genomics.

\section{Author contributions}

Conceptualization: S.M., R.V.-C., and D.G.J.; methodology: S.M., R.V.-C., A.K.B., M.T.B. and T.G.K.; investigation: S.M., R.V.-C., E.B., and B.J.K.; formal analysis: B.L.; writingoriginal draft: S.M. and D.G.J., writing-review and editing: R.V.-C., A.K.B., M.T.B., and T.G.K.; funding acquisition: M.T.B., T.G.K., and D.G.J., supervision: M.T.B., T.G.K., and D.G.J.

\section{Competing interests}

The authors declare no competing interests.

\section{Additional information}

Supplementary information is available for this paper at https://doi.org/10.1038/s41467019-12861-8.

Correspondence and requests for materials should be addressed to D.G.J.

Peer review information Nature Communications thanks Stefano Campaner and the other, anonymous, reviewer(s) for their contribution to the peer review of this work. Peer reviewer reports are available.

Reprints and permission information is available at http://www.nature.com/reprints

Publisher's note Springer Nature remains neutral with regard to jurisdictional claims in published maps and institutional affiliations.

\section{(c) (i)}

Open Access This article is licensed under a Creative Commons Attribution 4.0 International License, which permits use, sharing, adaptation, distribution and reproduction in any medium or format, as long as you give appropriate credit to the original author(s) and the source, provide a link to the Creative Commons license, and indicate if changes were made. The images or other third party material in this article are included in the article's Creative Commons license, unless indicated otherwise in a credit line to the material. If material is not included in the article's Creative Commons license and your intended use is not permitted by statutory regulation or exceeds the permitted use, you will need to obtain permission directly from the copyright holder. To view a copy of this license, visit http://creativecommons.org/ licenses/by/4.0/.

(C) The Author(s) 2019 